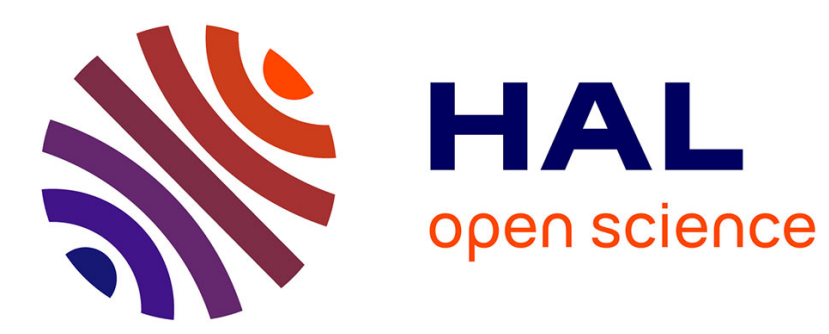

\title{
Modelling nanoparticle transport in dielectrophoretic microdevices using a FourierBessel series and applications for data analysis
}

\author{
D J Bakewell
}

\section{- To cite this version:}

D J Bakewell. Modelling nanoparticle transport in dielectrophoretic microdevices using a FourierBessel series and applications for data analysis. Journal of Physics D: Applied Physics, 2011, 44 (8), pp.85501. 10.1088/0022-3727/44/8/085501 . hal-00597848

\section{HAL Id: hal-00597848 \\ https://hal.science/hal-00597848}

Submitted on 2 Jun 2011

HAL is a multi-disciplinary open access archive for the deposit and dissemination of scientific research documents, whether they are published or not. The documents may come from teaching and research institutions in France or abroad, or from public or private research centers.
L'archive ouverte pluridisciplinaire HAL, est destinée au dépôt et à la diffusion de documents scientifiques de niveau recherche, publiés ou non, émanant des établissements d'enseignement et de recherche français ou étrangers, des laboratoires publics ou privés. 


\title{
Modelling nanoparticle transport in dielectrophoretic microdevices using a Fourier-Bessel series and applications for data analysis.
}

\author{
D J Bakewell \\ Department of Electrical Engineering and Electronics, University of Liverpool, UK. \\ Email: d.bakewell@liv.ac.uk
}

\begin{abstract}
A Fourier-Bessel series solution is derived that describes the dielectrophoretic-driven transport of nanoparticles in a microdevice. The solution assumes that the nanoparticles do not interact and is based on a linear Fokker Planck equation that includes the effects of thermal diffusion. The solution is applicable for a dielectrophoretic force that varies exponentially in the microdevice, such as, in the far field of planar interdigitated arrays. Important applications of the Fourier-Bessel solution are demonstrated that include simulation and system classification of nanoparticle movement under the action of weak and strong dielectrophoretic forces. Methods are demonstrated for the inverse process of estimating model parameters, such as the dielectrophoretic force, based on nanoparticle concentration data obtained experimentally. Data decomposition into separate spatial and temporal modes is demonstrated and Fourier transformation of the series solution yields a representation in the frequency domain. The frequency response predicted by transforming the time dependent FourierBessel solution indicates the presence of a DEP modulation bandwidth that concurs with observations of preliminary experiments.
\end{abstract}

Keywords: AC electrokinetics, dielectrophoresis, Fourier-Bessel series, modified diffusion equation, FokkerPlanck equation.

PACS: 87.85.Tu, 87.10.Ed, 87.85.Ox, 02.60.Cb

\section{Submitted to Journal of Physics D: Applied Physics}

\section{Introduction}

Dielectrophoresis (DEP) is a versatile noncontact electrokinetic method for controlling the movement of nanosize biomolecules and colloids in micro-environments. DEP nanoparticle transport processes have been intensely researched for lab-on-chip (LOC) type fabricated microdevices [1, 2], and for other diverse scientific and technological applications, including textile fabrics, scanning probe microscopes, biological manipulation tools, quantum dots, nano-circuit assembly and optical fluidics [3-13]. DEP is the movement of polarisable nanoparticles arising from the action of nonuniform electric fields [14]. It is often achieved by applying radio frequency electrical potentials to microfabricated electrodes immersed in low conductivity electrolyte. The application of DEP electrokinetics in micro-technologies means nanoscale nanoparticle movement needs to be modeled and measured quantitatively. Quantitative measurements of the DEP driven nanoscale transport by us [15-17] identified a number of time constants that raised questions about the theoretical basis of their origin and subsequently motivated this work on modelling.

The spatial-temporal distribution of nanosize nanoparticles moving under the action of a deterministic DEP force and stochastic Brownian thermal motion can be described by a linear, second order partial differential equation known as the modified diffusion equation or Fokker-Planck equation (FPE). The FPE is applicable for describing the trajectory of a single nanoparticle in an ensemble of identical microdevices, or an ensemble of non-interacting, mono-disperse nanoparticles in a microdevice. Integration over space and time of DEP-driven nanoparticle transport, using values for relevant dielectric and fluid parameters, is usually achieved by numerical methods, such as, Finite Element methods (FEMs). Considerable research has developed mathematical solutions that predict the electric field and DEP force in a microenvironment using methods that solve for the electric potential using Laplace's equation. These methods include Green's 
functions, conformal mapping, Fourier series, etc., [18-20]. Expressions for the spatially dependent DEP force are then typically applied to FEM simulations to predict nanoparticle position, often focusing on quasisteady states of the DEP transport process when the system has reached equilibrium.

The drawbacks of current methods, such as, FEMs are inaccuracy where the electric field is extremely inhomogeneous. In addition, although FEMs offer flexible simulation capabilities, they offer little insight into the mathematical structure of a FPE solution that is often useful for the development of new applications. Approximations for predicting DEP nanoparticle transport have also been developed, such as, the 'collection rate' $[17,21,22]$. The approximation considers DEP nanoparticle capture initially after the force has been switched 'on' when nanoparticle flux arising from thermally driven diffusion is considered to be negligible. Although these approximations have considerable utility, they are limited to using data for short time intervals and incur experimental error. To our knowledge there is very little research reported that considers DEPdriven nanoparticle transport in terms of mathematical models where integration in time yields generalised analytical, or closed-form, solutions.

This paper reports the development of a mathematical model for predicting DEP nanoparticle transport that enables analysis of the DEP-driven electrokinetic process, particularly with respect to time as a independent variable. Time-dependent DEP nanoparticle collection and release (after the DEP is switched off) from a surface is evaluated for strong and weak DEP forces. It is a major technical advance from a briefer report [23]. In that work a three dimensional (3D) chamber was simplified to 1D, and an infinite FourierBessel (FB) series solution to the FPE was found that described nanoparticle movement under the action of DEP. The DEP force was assumed to be generated by a horizontal planar electrode array and spatially decayed hyperbolically, i.e. a force that decreased with height above the array according to an inverse law. In this paper, nanoparticle transport in the far field of an array is considered where the DEP force decreases with height according to a spatial exponential profile. Planar arrays are common in LOC devices and it has been shown experimentally and theoretically that the DEP force in the far field, at least from an interdigitated planar array, decays exponentially [20,24]. The exponential spatial profile of DEP force is more challenging than earlier work because values for the general solution exist over the complex number field.

In addition to the prediction of the spatial-temporal evolution of nanoparticle concentration, figure 1(a), the paper is also a fundamental advance from previous work [23] in that it considers the 'inverse' process to simulation, i.e. estimation of transport model coefficients from experimental data, as shown in figure 1(b). The estimation process and exploitation of the FB series properties yields five important applications:

- classification of simulated or experimental data

- development of approximations for simulations, or data from experiments

- decomposition of experimental data into independent spatial and temporal components

- transformation of simulated or experimental data in space and time into alternative domains, e.g. from the time domain to the frequency domain

- representation of simulated or experimental data with functional elements that are familiar to engineers and physicists, e.g. low-pass filters.

The third application of combined estimation and decomposition is demonstrated using pseudo-experimental data. Given that estimation can be achieved, the other four applications are demonstrated using simulated data. The applications are described after the theory of nanoparticle transport is introduced and FB series solutions to the FPE model are developed. 


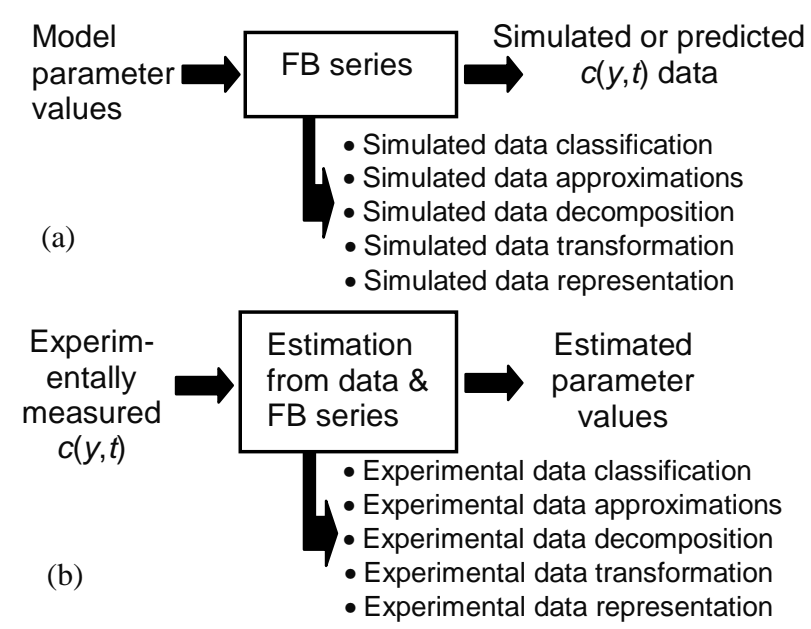

Figure 1. Application of Fourier-Bessel (FB) series for (a) prediction or simulation of spatial-temporal concentration $\mathrm{c}(y, t)$, and (b) estimation parameter values from experiments. Applications are as listed, see text for details.

\section{Theory: nanoparticle transport model}

A suspension of nanosize nanoparticles moving under the action of deterministic positive DEP ( $\mathrm{pDEP}$ ) force acting downwards in a microdevice is illustrated in Figure 2(a). The electric far-field approximation enables electrode end effects to be ignored for long and wide planar arrays so that the 3D nanoparticle transport is simplified to $1 \mathrm{D}$, as illustrated in figure 2(a).

\subsection{DEP force}

The DEP force arising from a planar array with side view shown in figure 2(a) is approximated as,

$$
F_{d e p}(y)=-k_{1} \exp \left(-k_{2} y\right), a \leq y \leq b
$$

where the constant $k_{2}=2 \pi /(w+g)$ is dependent on the electrode dimensions with (transverse) width, $w$, and inter-electrode gap, $g$. The force coefficient $k_{l}$ expresses the magnitude of the DEP force at the surface of the electrode plane. This means that if the lower boundary is set to the origin on the $y$ axis, $a=0$, then at $y=a$ the spatial maximum is, $F_{d e p}(0)=-k_{1}$. Using the same convention as previous work [20] the minus sign in front of $k_{l}$ indicates the force in this arrangement acts downwards towards the lower boundary for positive DEP (pDEP) where the numerical value is positive, $k_{1}>0$. Conversely, the force in figure 2(a) acts upwards for negative DEP (nDEP), so that $k_{l}<0$. The DEP force arising from a planar array with side view show in figure 2(a) is significantly stronger than the resultant force from gravity and buoyancy (sedimentation) for most of $y$ so the latter is ignored.

Theoretical values for $k_{l}$ are typically predicted by Maxwell-Wagner interfacial polarisation for spherical objects and are evaluated using the product of the Clausius-Mossotti factor, $f_{c m}$, nanoparticle volume, and the gradient of the magnitude of the electric field-squared, $\vec{\nabla}|\vec{E}|^{2}$ [14]. However, more recent work that compared DEP experimental data showed that the actual values of the DEP force, i.e. $k_{l}$, can be much less than predicted from simple interfacial theory [16]. The reasons for the DEP nanoparticle movement being compromised have not been fully elucidated although a number of effects have been investigated including fluid motion arising from AC electro-osmosis and other electrohydrodynamic effects [25]. There are other additional effects, e.g. the electric field needed for evaluating the DEP force was based on Laplace's equation. This is a simplification to Poisson's equation that takes into account the density of free charges, such as, ions 
in solution. Consequently, to accommodate the disparity between simple theory and experiment, $k_{l}$ is assigned as the effective DEP force coefficient that for the purposes of this paper can either be assigned, or estimated experimentally, $\hat{k}_{1}$, where ' $\wedge$ ' denotes estimate. This distinguishes $k_{l}$ from the all other coefficients, such as, $k_{2}$ that are assigned known values.

\subsection{Dielectrophoretic nanoparticle transport towards and away from a planar array}

The distribution of nanoparticles is over space and time is described by the nanoparticle concentration, $c(y, t)$ and the total number of nanoparticles, $N$, within the system remains constant throughout the course of the experiment, i.e. no nanoparticles enter or escape. The corresponding time-dependent nanoparticle concentration near the electrode array at $y=a$ is shown in figure 2(b). The size of the nanoparticles is on the nanoscale but they can be much larger; the key feature being that the stochastic effect of Brownian thermal motion is significant.

Before switching on the DEP force at $t<t_{\text {on }}$, figure 2(a)(i), the nanoparticles are uniformly distributed with concentration, $c_{i}$. After applying an AC potential to the electrodes, the action of the pDEP force causes downward nanoparticle movement, particularly near the electrode array where the DEP force is strong, figure 2(a)(ii). This rate of nanoparticle accumulation is often measured experimentally as the 'collection rate' that is usually measured up to a designated time, $t_{\mathrm{c}}$, in figure 2(b) $[17,26]$. As the concentration further increases near the array, DEP accumulation near the lower boundary results in a depletion layer that steadily rises towards the cap at $y=b$, as shown. The cap is located at height $h=b-a$ above the array, as shown. Eventually the DEP nanoparticle flux becomes balanced by thermally-driven diffusion, figure 2(a)(iii), and approaches steady state (SS), $t=t_{s s}$ with concentration, $c_{s s}$. Switching off the AC potential releases the nanoparticles since there is no longer any pDEP force to trap them, and they diffuse into the bulk medium, figure 2(a)(iv). On-off switching can be repeated, as reported for pDEP of DNA [16]. The difference between the SS and initial concentration is shown as $\Delta c=c_{s s}-c_{i}$.

It is convenient to predict or simulate DEP nanoparticle transport using concentration normalised with respect to $N$ in the closed system. The normalised concentration is the probability density function (pdf) $p(y, t)$ (per unit length),

$p(y, t)=c(y, t) / N$

and the axiomatic condition applies, $\int_{a}^{b} p(y, t) d y=1 \forall t, p(y, t) \geq 0$. In the following sections reference is made to the pdf or 'density'.

Experimental measurements of DEP process are subject to variation in the observed concentration between experiments. In later sections the process is considered in a systems context, therefore the relative change between the SS and initial density is introduced. It is the probability (or concentration) amplitude,

$A(y)=\frac{\Delta p(y)}{p(y, 0)}=\frac{c(y, \infty)-c(y, 0)}{c(y, 0)}=\frac{c(y, \infty)}{c(y, 0)}-1$

The relative quantity, $A$, can be determined using concentration data estimated from each DEP collection experiment and without requiring explicit knowledge of $N$. 

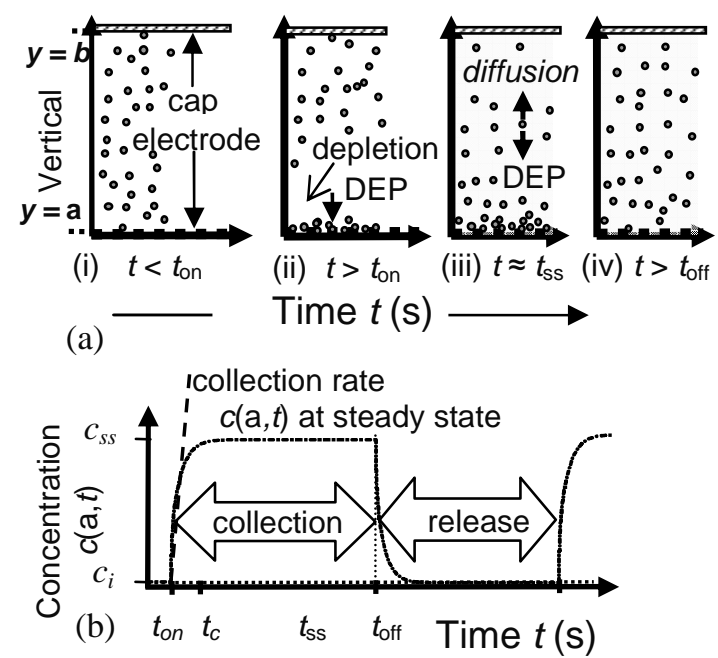

Figure 2. Nanoparticle collection under the action of pDEP force and release after the DEP force is switched off (a) cartoon showing nanoparticle distribution (side view) (b) pdf at the array as a function of time, $p(a, t)$, showing nanoparticle collection and release with corresponding times. See text for details.

\subsection{Fokker Planck equation}

The evolution of the density $p(y, t)$ is described by the forward FPE that combines nanoparticle flux contributions from DEP and thermal diffusion,

$$
\frac{\partial p(y, t)}{\partial t}=-\frac{\partial J(y, t)}{\partial y}=\underbrace{-\frac{1}{\zeta} \frac{\partial\left(p(y, t) F_{D E P}(y)\right)}{\partial y}}_{\text {DEP }}+\underbrace{\frac{k_{B} T}{\zeta} \frac{\partial^{2} p(y, t)}{\partial^{2} y}}_{\text {Diffusion }}
$$

The 'pdf flux' is $J(y, t)=\frac{p(y, t) F_{D E P}(y)}{\zeta}-\frac{k_{B} T}{\zeta} \frac{\partial p(y, t)}{\partial y}, k_{B} T$ is the Boltzmann temperature, and $\zeta$ is the dynamic drag coefficient. Eq. (2.4) does not lead to a stationary solution for deterministic forces, such as DEP, that markedly increase towards the surface. The DEP force, or potential, requires boundary conditions (BCs) and contrasts, for example, with nanoparticle movement in a parabolic potential [27] that yields a stationary solution without needing external BCs to be added. The FPE can be derived by considering mass continuity and applying Fick's first and second laws of diffusion. It is also derived by modelling single nanoparticle movement with a Langevin equation and considering this in an ensemble of microdevices, or as an ensemble of point-wise nanoparticles that are non-interacting and sufficiently dilute, e.g. below 1:1000 dilution of $1 \%$ solids. The ensemble of nanoparticle trajectories predicted by the Langevin equation can be integrated stochastically, or by other methods, to yield an FPE [27, 28].

\subsection{Boundary conditions (BCs) and initial condition (IC)}

The magnitude of positive DEP force is very large near the electrode array (or boundary), thereby strongly attracting nearby nanoparticles towards it, as shown in figure 2(a). The actual electrode array surface, however, is impenetrable to nanoparticles; so although the nanoparticles are increasingly attracted as they approach the boundary, their movement is ultimately reversed. Thus, the electrode array forms the lower, reflecting Boundary Condition (BC) at $y=a$. The upper, reflecting $\mathrm{BC}$ is formed by the chamber cap at $y=b$, 
as shown. The two impenetrable barriers mean the nanoparticle flux $J$ defined in (2.4) is zero at $y=a$ and $y=$ b. This yields two BCs for the 1D model,

$$
J(a, t)=0=J(b, t) \forall t
$$

Since most biological nanoparticles of interest exhibit a mass density similar to the electrolyte so that gravity can be neglected, a useful Initial Condition (IC) at the start of a DEP nanoparticle collection experiment is the uniform distribution. Applying (2.1), the IC is

$$
\int_{a}^{b} p(y, t) d y=1, \text { at } t=0 \Rightarrow p(y, 0)=\frac{1}{b-a}=\frac{1}{h}
$$

\section{Solutions to the FPE model}

The FPE given by (2.4), combined with the BCs and IC, is integrated this section to obtain the steady state and time dependent densities.

\subsection{FPE steady state solution}

Setting the lhs to zero in the FPE (2.4), integrating and applying the zero fluz BCs (2.5) and axiomatic relation (2.2), yields the SS solution for the density,

$$
p_{s s}(y)=\left\{\begin{array}{ll}
\frac{k_{2} \exp \left(\lambda e^{-k_{2} y}\right)}{E i\left(\lambda e^{-k_{2} a}\right)-E i\left(\lambda e^{-k_{2} b}\right)}, & \lambda \neq 0 \\
\frac{1}{b-a}, & \lambda=0
\end{array}\right\}
$$

where $\lambda=\frac{k_{1}}{k_{2} k_{B} T}, E i$ denotes the exponential integral function defined in terms of the Cauchy Principle Value (CPV) that is readily calculated in Matlab ${ }^{\mathrm{TM}} 7.8$ (Mathworks Inc., MA, USA), and other remaining symbols have been previously defined. As expected, the SS solution shows dependence on the ratio of the DEP force coefficient with respect to the thermal temperature, $k_{1} / k_{B} T$ and it is understood $p_{s s}(y)=p(y, t \rightarrow \infty)$.

The relationship between density and DEP force is important for characterising DEP transport. Four plots of the pdf amplitude, $A$, at the lower boundary, $y=a$, versus $k_{l}$ are shown in figure 3 . The amplitude was calculated from equations (2.3) and (3.1). The four plots correspond to combinations of narrow and wide electrode widths, $w+g=10 \mu \mathrm{m}$ and $40 \mu \mathrm{m}$, and low and high chamber heights, $h=40 \mu \mathrm{m}$ and $200 \mu \mathrm{m}$. The four trends of $A\left(k_{1}\right)$ show a linear relationship on a log-log scale for weak DEP forces up to about $k_{1}=1 \mathrm{fN}$. Above this value a transition occurs as stronger DEP force is responsible for accumulating almost all nanoparticles, that were initially uniformly distributed within the chamber, against the lower boundary. There occurs an almost exponential increase until about $k_{1}=10 \mathrm{fN}$ and the trends return to a linear trend. The proximity of the $A\left(k_{1}\right)$ plots to each other arises from the fact that $N$ is constant across all four simulations and shows expected characteristics. Experimentally this corresponds to diluting the suspension by adding medium (e.g. water) to fill the volume as the height is increased from $40 \mu \mathrm{m}$ to $200 \mu \mathrm{m}$. That is, for $k_{1}$ up to $1 \mathrm{fN}$, the plots are shown to pair according to the array geometry: $w+g=10 \mu \mathrm{m}$ plots practically overlap and the $40 \mu \mathrm{m}$ pair likewise. This is because the localised DEP force has a dominant effect for weak DEP forces and the height has little influence. Conversely, at strong DEP forces above $k_{1}=20 \mathrm{fN}$, the plots pair according to the 
chamber height, i.e. $h=40 \mu \mathrm{m}$ and $200 \mu \mathrm{m}$ are paired. This is due to the strong DEP forces causing largescale nanoparticle transport from the top to the bottom of the chamber, hence, collecting nearly all of the nanoparticles. The key point is the transition of the pairing at about $20 \mathrm{fN}$ that distinguishes weak from strong DEP regimes, as shown.

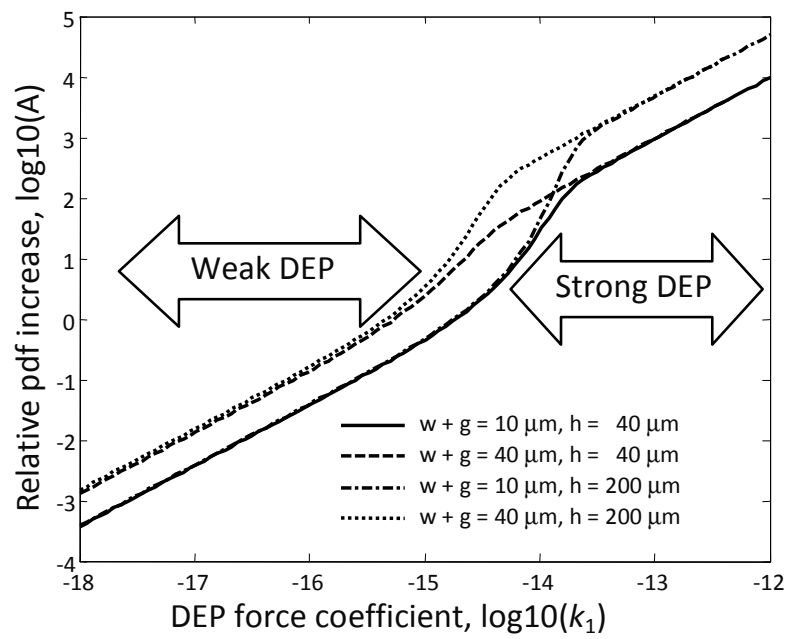

Figure 3. Relative probability density increase $\log _{10}(\mathrm{~A})$ versus $\log 10\left(k_{l}\right)$ plots show the transition between weak DEP and strong DEP regimes.

\subsection{FPE time dependent general solution: Fourier-Bessel series}

Expanding the density $p(y, t)$ in a series product of separate functions $g(y)$ and $h(t)$,

$$
p(y, t)=\sum_{m=0}^{\infty} g_{m}(y) h_{m}(t)
$$

Substituting (3.2) into (2.5) yields two separate, linear Ordinary Differential Equations (ODEs). The first ODE has unity order with a General Solution (GS) for each eigenmode $m$,

$$
h_{m}(t)=e^{-\rho_{m}^{2} t} \equiv e^{-\frac{t}{\tau_{m}}}
$$

where $\rho_{m}$ is an arbitrary, real, integration constant. The re-scaled, reciprocal of the eigenvalue squared is often used interchangeably, and is the eigenmode time constant, $\tau_{m}=1 / \rho_{m}^{2}$. The second ODE is

$g_{m}^{\prime \prime}(y)-\frac{\zeta}{k_{B} T} g_{m}^{\prime}(y) v(y)+\left(\rho_{m}^{2}-v^{\prime}(y)\right) \frac{\zeta}{k_{B} T} g_{m}(y)=0$

where the primes denote derivatives with respect to $y$, and $v(y)=F_{D E P}(y) / \zeta$ is the DEP drift velocity. A GS to (3.4) is found using listed formulae [29] or Maple ${ }^{\mathrm{TM}} 13$ (Waterloo, Canada),

$$
g_{m}(y)=e^{c(y)}\left[C_{1_{m}} I^{\dagger}\left(\alpha_{m}, \kappa(y)\right)+C_{2_{m}} K^{\dagger}\left(\alpha_{m}, \kappa(y)\right)\right]
$$


where $I^{\dagger}\left(\alpha_{m}, \kappa(y)\right)=I\left(\alpha_{m}, \kappa(y)\right)+I\left(-\alpha_{m}^{*}, \kappa(y)\right) \quad$ and $\quad K^{\dagger}\left(\alpha_{m}, \kappa(y)\right)=K\left(\alpha_{m}, \kappa(y)\right)-K\left(\alpha_{m}^{*}, \kappa(y)\right)$ are linearly independent combinations of modified Bessel functions of the first and second kind. In (3.5) $\alpha_{m}=\frac{1}{2}+\frac{i \beta_{m}}{k_{2}}, \beta_{m}=\rho_{m} \sqrt{\frac{\zeta}{k_{B} T}}$ denotes complex order, $\kappa(y)=\frac{k_{1} e^{-k_{2} y}}{2 k_{2} k_{B} T}=\frac{\lambda}{2} e^{-k_{2} y}$ is the real argument, $i=\sqrt{-1}$ , and $*$ denotes complex conjugate. Modified Bessel functions with complex order are not always supported in some computing languages, such as, Matlab ${ }^{\mathrm{TM}} 7.8$, however, they can be numerically evaluated using established algorithms [30]. The function common to both modified Bessel functions and independent of the eigenmode, $m$, is $c(y)=\frac{k_{1} e^{-k_{2} y}}{2 k_{2} k_{B} T}-\frac{k_{2} y}{2}=\kappa(y)-\frac{k_{2} y}{2} ; \rho_{m}, C_{1_{m}}$ and $C_{2_{m}}$ are arbitrary integration constants.

Our interest lies in the real parts of the solution that are sufficient to describe the translational motion of nanoparticle transport within the chamber. Further, $\operatorname{Re}\left\{K^{\dagger}\right\}=0$ where $\operatorname{Re}\{.\}=$.0 denotes Real part. Hence (3.5) is re-cast ansatz,

$$
g_{m}(y)=C_{m} e^{c(y)} \underbrace{\left.\operatorname{Re}\left\{I^{\dagger}\left(\alpha_{m}, \kappa(y)\right)\right\}-D_{m} \operatorname{Im}\left\{K^{\dagger}\left(\alpha_{m}, \kappa(y)\right)\right\}\right]}_{\tilde{g}_{m}(y)}
$$

where $\operatorname{Im}\{.\}=$.0 denotes Imaginary part, and the integration constants are real and imaginary, $C_{m} \in R$ and $D_{m} \in I$. To evaluate the three sets of integration constants, two BCs and an IC are imposed. The probability flux in (2.4) and (2.5) is written as an eigenfunction expansion and the derivatives of the modified Bessel functions in (3.6) with respect to $y$ are readily evaluated using recursion formulae [29]. Mathematical expressions can be verified by 'pencil-and-paper' and simplified by recognising, $\alpha-1=-\alpha^{*}$. The zero flux $\mathrm{BCs}$ are applied to the GS, thus yielding two simultaneous equations in matrix form,

$$
\left(\begin{array}{cc}
\operatorname{Re}\left\{\tilde{I}\left(\rho_{m}, a\right)\right\} & \operatorname{Im}\left\{\tilde{K}\left(\rho_{m}, a\right)\right\} \\
\operatorname{Re}\left\{\tilde{I}\left(\rho_{m}, b\right)\right\} & \operatorname{Im}\left\{\tilde{K}\left(\rho_{m}, b\right)\right\}
\end{array}\right)\left(\begin{array}{c}
1 \\
-D_{m}
\end{array}\right)=\left(\begin{array}{l}
0 \\
0
\end{array}\right), \rho_{m} \neq 0, C_{m} \neq 0
$$

where $\tilde{I}\left(\rho_{m}, y\right)=i \beta_{m} e^{c(y)}\left[I\left(\alpha_{m}, \kappa\right)-I\left(-\alpha_{m}^{*}, \kappa\right)\right], \tilde{K}\left(\rho_{m}, y\right)=i \beta_{m} e^{c(y)}\left[K\left(\alpha_{m}, \kappa\right)+K\left(-\alpha_{m}^{*}, \kappa\right)\right]$ and it is understood $\alpha_{m} \equiv \alpha\left(\rho_{m}\right)$ and $\kappa \equiv \kappa(y)$ defined in (3.5). Finding the roots of the matrix determinant, $\Delta$, that is a nonlinear function,

$$
\left\{\rho_{m}: \Delta(\rho)=\operatorname{Re}\{\tilde{I}(\rho, b)\} \operatorname{Im}\{\tilde{K}(\rho, a)\}-\operatorname{Re}\{\tilde{I}(\rho, a)\} \operatorname{Im}\{\tilde{K}(\rho, b)\}=0\right\}
$$

yields the eigenvalues, $\rho_{m}$. Evaluation of this first set of $m$ integration constants enables evaluation of the second set of integration constants given by,

$$
D_{m} \equiv \frac{\operatorname{Re}\left\{\tilde{I}\left(\rho_{m}, b\right)\right\}}{\operatorname{Im}\left\{\tilde{K}\left(\rho_{m}, b\right)\right\}}
$$

To evaluate the remaining set of integration constants, $C_{m}$, an orthogonal basis over the interval $\{y: a \leq y \leq b\}$ is established using the backward FPE and techniques [27] that use the SS condition (3.1). Applying the IC (2.6) and steps in Appendix A leads to 
$C_{m}=\frac{\int_{a}^{b} p(y, 0) e^{c(y)} \tilde{g}_{m}(y) e^{-\lambda e^{-k_{2} y}} d y}{\int_{a}^{b} e^{2 c(y)} \tilde{g}_{m}(y)^{2} e^{-\lambda e^{-k_{2} y}} d y}=\frac{\frac{1}{b-a} \int_{a}^{b} e^{c(y)} \tilde{g}_{m}(y) e^{-\lambda e^{-k_{2} y}} d y}{\int_{a}^{b} e^{2 c(y)} \tilde{g}_{m}(y)^{2} e^{-\lambda e^{-k_{2} y}} d y}$

where $\tilde{g}_{m}(y)$ defined in (3.4) is evaluated in terms of $D_{m}$ and $\rho_{m}$. An example plot of the Bessel matrix determinant (3.7) that is solved to yield eigenvalues is shown in figure 4. The determinant exhibits an oscillatory behaviour with near-periodic roots that are numerically evaluated using the root finding algorithm in Matlab ${ }^{\mathrm{TM}} 7.8$.

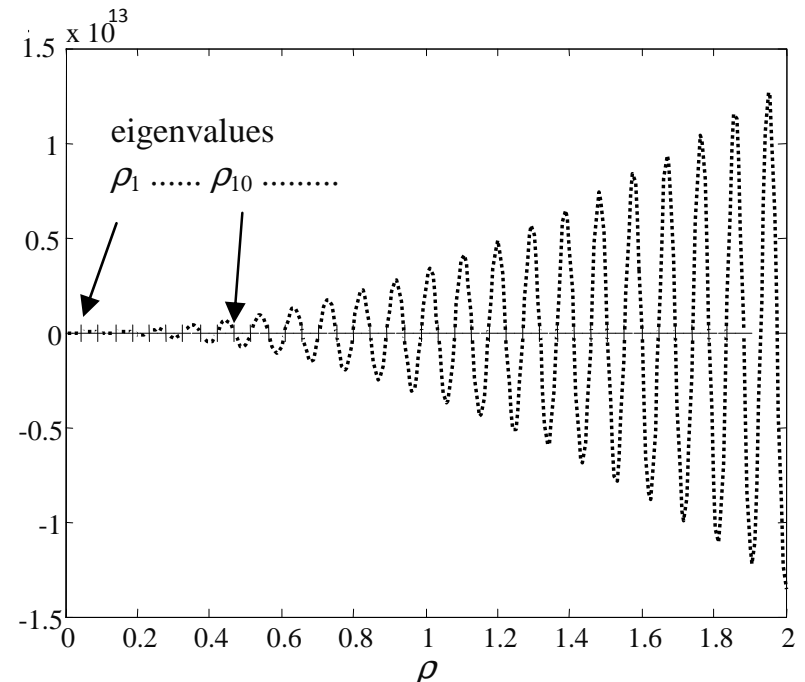

Figure 4. Determinant $\Delta$ as a function of $\rho$ showing roots yielding eigenvalues $\rho_{m}$.

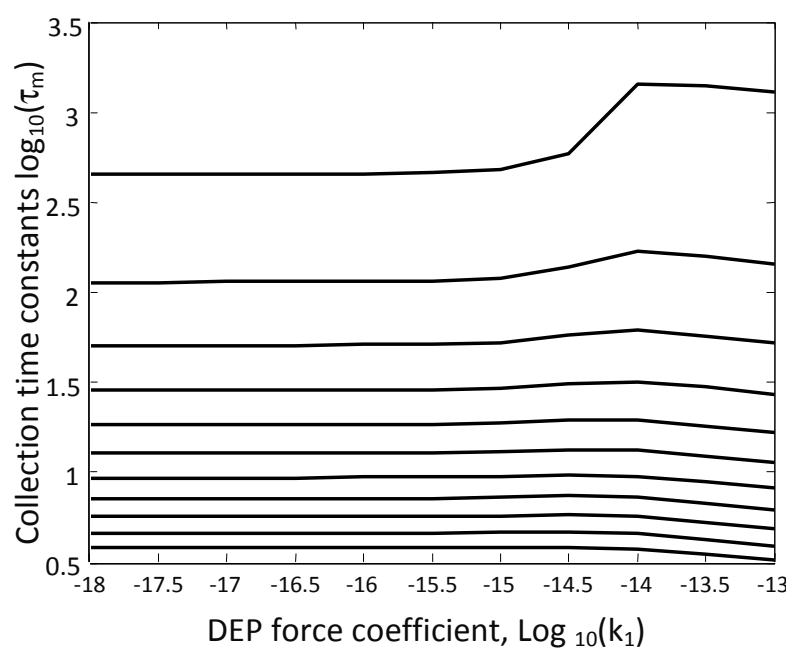

Figure 5. Plot of eigenmode time constants $\tau_{m}$ versus $k_{l}$ for $h=100 \mu \mathrm{m}$

The eigenvalues are re-scaled as the modal time constants defined in (3.3). The dependence of the time constants on the DEP force $k_{l}$ is plotted in figure 5. They exhibit only a gradual change as the DEP force ranges over many orders of magnitude up to about 1 femto-Newton (fN). For higher DEP forces ranging from 1 to $100 \mathrm{fN}$, the eigenmodal time constants increase then decrease, particularly the first mode, $m=1$. Simplifying the Bessel functions for asymptotically small values of the arguments as $k_{1} \rightarrow 0$, the eigenvalues can be shown to converge to limiting values that correspond to the case of diffusive transport, i.e. absence of DEP force,

$\rho_{m}=\frac{\pi m}{h} \sqrt{\frac{k_{B} T}{\zeta}}$

where all symbols have been previously defined. These values were used to initialize the determinant root finding algorithm for generating eigenvalues for collections with a wide range of DEP forces. Combining (3.3) and (3.11) confirms the general relation that the average time $t$ for a nanoparticle to diffuse over a length $l$ is proportional to the square of that length, $t \propto l^{2}$. 


\subsection{FPE predictions of DEP nanoparticle collection followed by release.}

A typical DEP nanoparticle response experiment often involves nanoparticle collections onto the electrodes until the system is at quasi-SS. The DEP process is then switched off and the nanoparticles move away from the electrode boundary under the action of thermal diffusion, and are released into the bulk medium.

\subsubsection{DEP nanoparticle collection assuming initial uniform concentration}

The particular solution (PS) for nanoparticle collection is found by substituting the (3.3), (3.6) and integration constants $\rho_{m}, D_{m}$ and $C_{m}$, into (3.2). The density is described by a Fourier-Bessel series, [31-33] and it is verified in Appendix B that the $m=0$ term of the eigenfunction expansion is the DEP SS term given by (3.1). Therefore, the spatial-temporal density for DEP nanoparticle collection is written

$$
p(y, t)=\underbrace{p_{s s}(y) u(t)}_{\text {steady state }}+\sum_{m=1}^{\infty} \underbrace{C_{m} e^{c(y)} \tilde{g}_{m}(y)}_{\text {spatial }} \underbrace{e^{-\frac{t}{\tau_{m}}} u(t)}_{\text {temporal }}
$$

where $\tilde{g}_{m}(y)=\operatorname{Re}\left\{I^{\dagger}\left(\alpha_{m}, \kappa(y)\right)\right\}-D_{m} \operatorname{Im}\left\{K^{\dagger}\left(\alpha_{m}, \kappa(y)\right)\right\}, u(t)$ is the Heaviside unit step function [34] that invokes causality. Typically, the spatial and temporal terms are convergent and the infinite FB series is evaluated by truncating $m$ to a finite integer, $m_{c}$, where the subscript $c$ denotes collection process. Truncation of the series will yield a computational error so the selection of $m_{c}$ is a trade-off between computation time, accuracy needed for the application and convergent properties of the series. Hence,

$$
p(y, t) \cong p_{s s}(y) u(t)+\sum_{m=1}^{m_{c}} C_{m} e^{c(y)} \tilde{g}_{m}(y) e^{-\frac{t}{\tau_{m}}} u(t)
$$

Typically, values of $m_{c}$, ranging from 15 to 40, gave a satisfactory computation time and accuracy trade-off.

An example surface plot of the collection pdf, $p(y, t)$, is shown in figure 6 for parameter values $k_{l}=1$ $\mathrm{fN}, w+g=20 \mu \mathrm{m}, a=0 \mu \mathrm{m}, h=100 \mu \mathrm{m}$, and $k_{B} T / \zeta=2.24 \times 10^{-12} \mathrm{~m}^{2} \mathrm{~s}^{-1}$ with a uniform distribution for the IC. The value of the thermal temperature and dynamic drag coefficients correspond to experimental values for $216 \mathrm{~nm}$ diameter spheres collecting in water at $25^{\circ} \mathrm{C}$. These values were used in experiments $[17,26]$ and are used for all simulations in this paper unless otherwise stated. The simulation used 40 eigenvalues, $m_{c}=40$, starting at $t=0$ seconds. A consequence of truncating the series to 40 terms is that $p(y, 0)$ was not exactly uniform near the lower boundary, so it remedied by setting $p(y, 0)=1 / \mathrm{h}$. Elsewhere the error arising from the series truncation was found to be negligible.

In figure 6 , the density, $p(y, t)$, shows a progressive increase near the lower impenetrable barrier at $y=a=0 \mu \mathrm{m}$ in response to the pDEP force that attracts the nanoparticles towards the boundary. As the nanoparticles move towards the lower boundary from above, a region of nanoparticle depletion occurs that progressively widens and shifts towards the upper boundary at $100 \mu \mathrm{m}$, as shown. The nanoparticle accumulation near the lower boundary is initially rapid due to the DEP driven nanoparticle flux that dominates over diffusion. The decreasing rate of accumulation after the DEP force is applied is typical of a diffusionlimited transport process that tends to SS. The plot was independently checked with a numerical FEM platform FlexPDE ${ }^{\mathrm{TM}} 2.15$ (PDE Solutions, USA) and showed very good agreement (see publisher's website for supplementary data). 


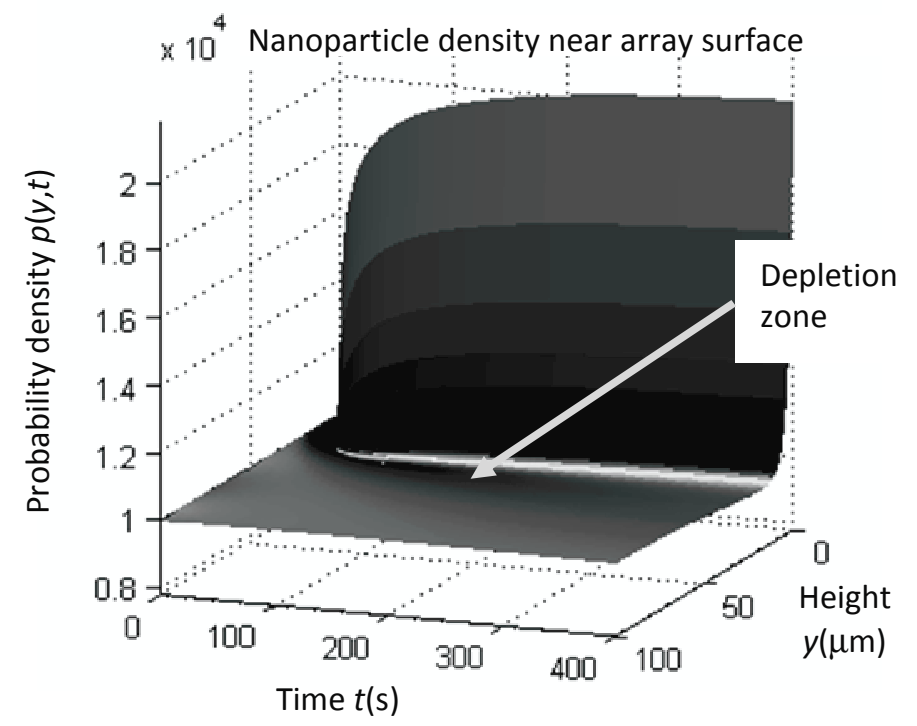

Figure 6. Probability density $p(y, t)$ in $100 \mu \mathrm{m}$ chamber showing DEP driven nanoparticle collection at array surface and depletion zone that disperses with time. A higher resolution in colour is available as supplementary data.

\subsubsection{Nanoparticle release following collection at steady state}

Switching off the DEP force causes the release of the nanoparticles from the array under the action of diffusion. Mathematically, setting $k_{1}=0$ allows the second order ODE (3.4) to be simplified. Using the similar methods described above for the FB series yields an infinite trigonometric Fourier series solution that can be truncated for computation,

$$
p_{r}(y, t)=\frac{1}{h}+\frac{2}{h} \sum_{m=1}^{m_{r}}\left[\int_{a}^{b} p_{r}(y, 0) \cos \left(\frac{\pi m(y-a)}{h}\right) d y\right] \cos \left(\frac{\pi m(y-a)}{h}\right) e^{-\rho_{m}^{2} t}
$$

It is understood $\rho_{m}^{2}=\frac{\pi^{2} m^{2}}{h^{2}} \frac{k_{B} T}{\zeta}$ for the case of diffusion that exhibits a square-law dependence on $m$. To avoid ambiguity with (3.13), the ' $r$ ' subscript is introduced for the density and maximum summation integer, $m_{r}$, to denote the process of nanoparticle release. As with the nanoparticle collections, the selection of $m_{r}$ is a trade-off between computation time, accuracy needed for the application and convergence properties of the series. Typically, $m_{r}=200$ eigenvalues yielded an acceptable performance. Assuming that the IC is the previous SS condition given by $(3.1), p_{r}(y, 0)=p(y, \infty)$, enables evaluation of the PS, (3.14). The integral in (3.14) was calculated numerically and integrating-by-parts and using cosine and sine integral functions [29] also gave the same results.

\section{Applications of the Fourier-Bessel series}

The Fourier-Bessel series enables decomposition of the nanoparticle concentration into separate, orthogonal temporal and spatial modes. These properties are used to develop and demonstate five key applications: classification, approximation, parameter estimation and decomposition of experimental data, transformation and representation. 


\subsection{Classification}

The temporal DEP response predicted by the FB series can used to classify nanoparticle transport process. Simulations of nanoparticle collections generated by the FB series, are shown in figure 7(a) for weak DEP forces $\left(k_{l}=0.1 \mathrm{fN}\right)$ and strong DEP force $\left(k_{l}=10 \mathrm{fN}\right)$. They are also plotted for cases of low and high chamber heights, $h=20$ and $100 \mu \mathrm{m}$. Since the probability densities vary over two orders of magnitude with respect to $k_{l}$, the time profiles of the pdfs have been normalised (denoted by subscript ' $n$ '),

$$
p_{n}(t)=\left[p(a, t)-p_{0}\right] /\left[p_{s s}-p_{0}\right]
$$

where $p_{0}=p(a, 0)$ and $p_{s s} \equiv p(a, \infty)$. Consequently, irrespective of $k_{1}, k_{2}$ and $h$ values, $0 \leq p_{n}(t) \leq 1$ so that all the densities time profiles can be compared in one figure. As with the SS characteristics described in section 3.1, $N$ is constant across all four simulations. A single point parameter to quantify the response is the DEP 'on' time constant, $\tau_{o n}$, which is defined as the time to reach $63 \%$ final SS with respect to initial value. Values corresponding to each of four time profiles are listed in Table 1. After the collection process has reached SS (or quasi-SS) the DEP force is switched off. Figure 7(b) shows the simulated time dependent nanoparticle release. Associated times, $\tau_{\text {off }}$ are defined as the time to reach $63 \%$ final SS and the nanoparticles become uniformly distributed - equibrating to the IC for collection.
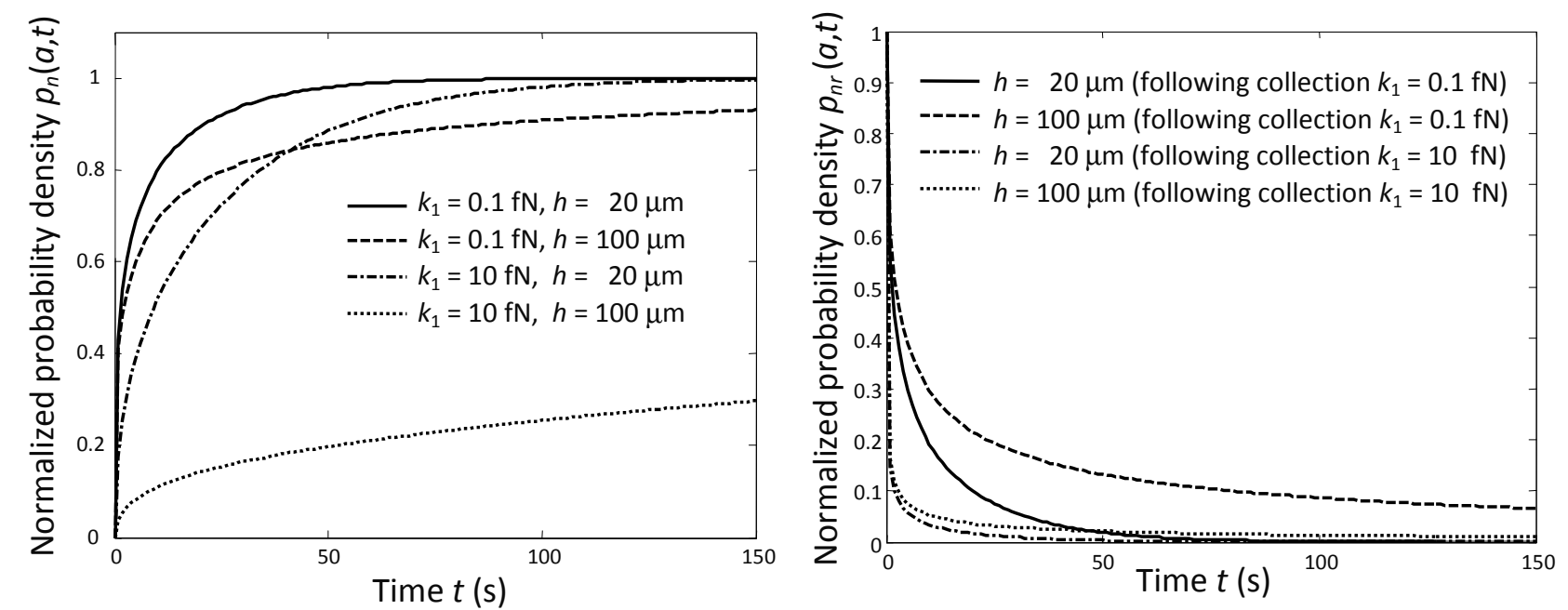

Figure 7. Nanoparticle time profiles for (a) DEP collection onto lower boundary (electrode array) at $y=a$, (b) nanoparticle release solely by diffusion into bulk solution. The profiles were independently verified with numerical simulations.

Table 1. Dependency of nanoparticle concentration response times on microdevice height and DEP strength.

\begin{tabular}{|c|c|c|c|c|}
\hline \multicolumn{2}{|c|}{ DEP force (fN) } & Height $\boldsymbol{h}(\boldsymbol{\mu m})$ & $\boldsymbol{\tau}_{\text {on }}(\mathrm{s})$ & $\boldsymbol{\tau}_{\text {off }}(\mathrm{s})$ \\
\hline \multirow{2}{*}{ Weak } & 0.1 & 20 & 3.6 & 3.2 \\
\cline { 2 - 5 } & 0.1 & 100 & 6.4 & 5.8 \\
\hline \multirow{2}{*}{ Strong } & 10 & 20 & 17 & 0.12 \\
\cline { 2 - 5 } & 10 & 100 & $>150$ & 0.14 \\
\hline
\end{tabular}

Keeping in mind the trends predicted SS in section 3.1, the results in Table I and figure 7(a) and 7(b) distinguish microdevice nanoparticle transport behaviour under different DEP strengths. They indicate that weak effective DEP forces collect only a minor proportion of the nanoparticles in short time durations, $\tau_{\text {on }} \cong \tau_{\text {off }}$ with $h$ not being very influential, and represent a system perturbation. Conversely, strong effective DEP forces collect a major proportion of the nanoparticles where almost all of the nanoparticles have collected on the electrode array, thus leaving elsewhere in the chamber depleted. The process takes longer to 
collect with the nanoparticle transport strongly dependent on $h, \tau_{o n} \gg \tau_{\text {off }}$, and therefore represents a state transition.

\subsection{Approximations}

Evaluations of the spatial and temporal terms show that for the weak DEP force, $g_{m}(a)-g_{m_{r}}(a)$ and $\tau_{m} \tau_{m_{r}}$. This means that, to a good approximation, the time response profiles complement each other and this is evident by both plots for the case, $k_{l}=0.1 \mathrm{fN}$, as shown in figure 7(a) and 7(b). Hence, the temporal relationship of nanoparticle collections and release can be approximated,

$$
p_{n}(a, t)+p_{n_{r}}(a, t+T) \cong 1 \text { for } 0 \leq t \leq T
$$

where $T$ denotes the period the DEP force is switched 'on'. In this example, $T$ is the same as the 'off' period (150 seconds). This approximation is useful for system functional block evaluation and representation described later in section 4.5.

Variations in the DEP force, given by small perturbations $\delta k_{1}$ give rise to changes in the density and these variations in nanoparticle concentrations are observed experimentally. In contrast, the plots of eigenmode time constants in figure 5 , with respect to $k_{l}$, shows they remain essentially constant for low DEP forces. Rewriting the FB series (3.13) in terms of signal amplitude, the temporal terms can be approximated, $h_{m}(t)=h\left(t, \rho_{m}+\delta \rho_{m}\right) \cong h\left(t, \rho_{m}\right)=\exp \left(-t / \tau_{m}\right)$, so that

$A\left(y, t, k_{1}+\delta k_{1}\right) \cong A_{s s}\left(y, k_{1}+\delta k_{1}\right)+\frac{1}{p_{0}} \sum_{m=1}^{\infty} g_{m}\left(y, k_{1}+\delta k_{1}\right) e^{-\frac{t}{\tau_{m}}}$

Where, as before, the subscript 'ss' denotes SS and $p_{0}=\frac{1}{b-a}$ is the IC for collection. Applying a first order Taylor series approximation

$$
A_{s s}\left(y, k_{1}+\delta k_{1}\right) \cong A_{s s}\left(y, k_{1}\right)+\delta k_{1} \partial_{k_{1}} A_{s s} \cong s_{A} A_{s s}
$$

where it can be shown

$$
\partial_{k_{1}} A_{s s}=\frac{\partial A_{s s}}{\partial k_{1}}=\left[\frac{p(b)-p(a)}{k_{1}}+\frac{e^{-k_{2} y}}{k_{B} T}\right] \frac{p(y)(b-a)}{k_{2}}
$$

and the amplitude scaling function is $s_{A}=1+\delta k_{1} \partial_{k_{1}} A_{s s} / A_{s s}$. It is understood $A_{s s}=A_{s s}\left(y, k_{1}\right)$ and the approximation is valid provided $\partial_{k_{1}} A_{s s} / A_{s s}$ is relatively smooth and well behaved. Suppose nanoparticle collection at the array is considered, $y=a$, and the series terms $g_{m}\left(a, k_{1}+\delta k_{1}\right) \cong s_{p} g_{m}\left(a, k_{1}\right)$ are also approximated in the same way, then 


$$
\begin{aligned}
A\left(a, t, k_{1}+\delta k_{1}\right) & \cong s_{A} A_{s s}\left(a, k_{1}\right)+\sum_{m=1}^{\infty} s_{A} g_{m}\left(a, k_{1}\right) e^{-\frac{t}{\tau_{m}}} \\
& \cong s_{A} A\left(a, t, k_{1}\right)
\end{aligned}
$$

That is the time profile for the density due to the perturbed DEP force can be rescaled. An example time profile is shown in figure 8(a) for the case $a=0 \mu \mathrm{m}$, electrode dimensions $w+g=20 \mu \mathrm{m}, h=100 \mu \mathrm{m}$ with $k_{l}$ $=1 \mathrm{fN}$. The original time profile is labelled, $A\left(a, t, k_{1}\right)$. Comparing the perturbed $A\left(a, t, k_{1}+\delta k_{1}\right)$ and the approximation $s_{A} A\left(a, t, k_{1}\right)$ shows they agree very closely. That is, the profile for the incremented DEP force can be obtained simply by rescaling or stretching the original; in this case by $14 \%$. Examination of the spatial eigenmode functions in figure 8(b) shows that $g_{m}\left(a, k_{1}+\delta k_{1}\right) \cong s_{A} g_{m}\left(a, k_{1}\right)$ is generally valid for all eigenvalues shown, though the approximation for the first few could be further improved.

The plots of eigenvalue time constants in figure 5 at stronger DEP forces above $k_{1}>1 \mathrm{fN}$ shows that several, including the first dominant eigenmode, $m=1$, increase before decreasing. The overall time response tends to be slower and is expected since the stronger force is transporting a large proportion of nanoparticles towards the electrodes in the chamber volume. Increases in $k_{l}$ in this moderately strong operation regime causes practically all nanoparticles to accumulate towards the electrodes. Further increases represent very strong DEP forces that consequently take less time to transport the nanoparticles, so the overall time response is faster. The dependence of the series with respect to the eigenvalues is likely to make an approximation in this higher DEP force regime more complicated, albeit the changes are shown to be less than an order of magnitude.
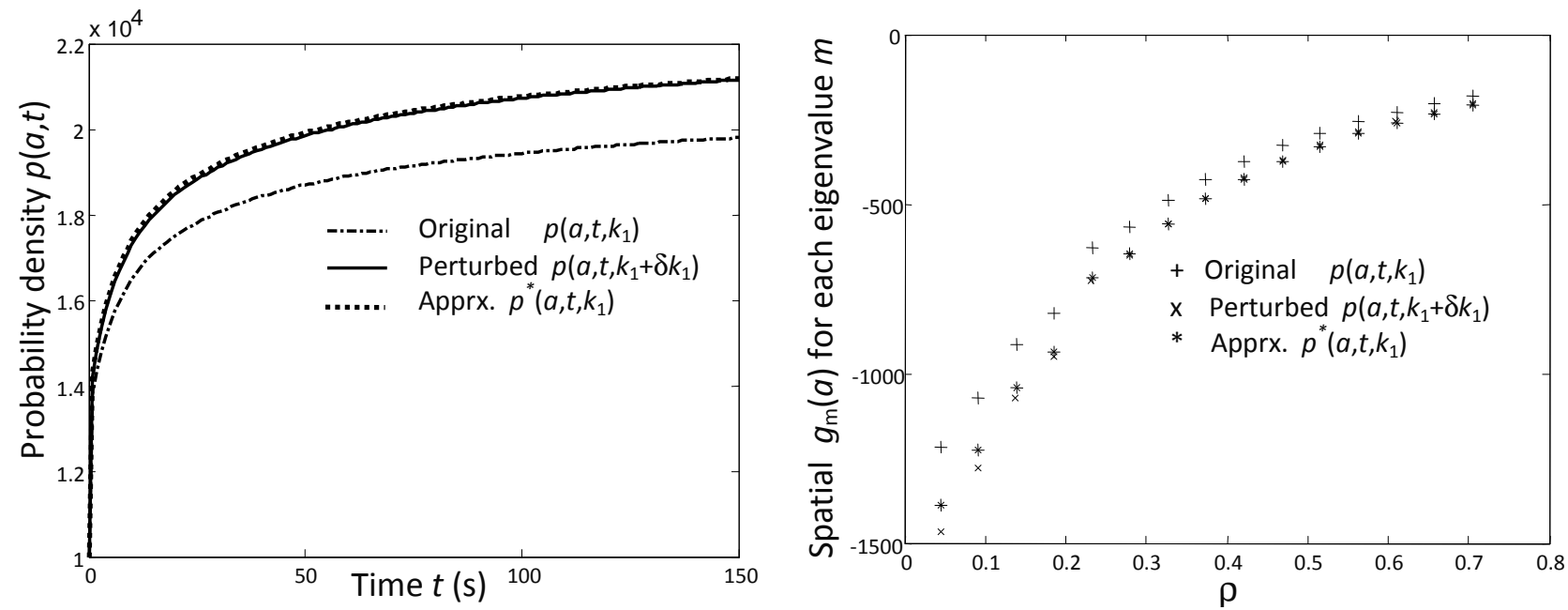

Figure 8. Perturbation and approximation to perturbation (a) time profiles (b) spatial eigenvalues at $y=a$

\subsection{DEP force estimation and decomposition of time response}

The FB solution (3.13) is a composite model entailing a sum of products of spatial and temporal components. Since the product of each FB series mode is explicit, in terms of using the FB series for simulation, decomposition is trivial. However, the inverse process of estimating parameter values, e.g. $k_{l}$, from nanoparticle concentration data, $c(y, t)$, and decomposing the spatial and temporal components is nontrivial and is important for analysis and understanding. In this section estimation and decomposition are combined and the principles are demonstrated using DEP collection data that mimics experiment. In terms of technological applications, the concentration data would be obtained from experiments or, conceivably, as surveillance measurements of an in situ DEP microdevice. Since decomposition of data with unknown parameter values itself, requires initial starting values for parameters, such as, $k_{l}$, there are two approaches. 


\subsubsection{Multiple eigenmode estimation and decomposition}

The first approach is a straightforward fit of the FB composite time profile (3.13) to the data using initial estimates of the parameter values. The fit is optimised, and the evaluated eigenmode terms can be displayed, thus decomposing imputed data. To demonstrate the principle of estimation, an algorithm was developed for finding an initial estimate of $k_{1}$ from the IC and SS (initial step), then refined with data from the FB time profile to obtain an improved estimation (refinement step). The two-step estimation scheme is shown in figure 9 and the time profile example of noisy data is shown in figure 10. The noisy data comprises the FB simulation (3.13) added with Gaussian noise that typified measurements of DEP collection experiments,

$\underbrace{p_{d}(a, t)}_{\text {data }}=\underbrace{p(a, t)}_{\text {true }}+\underbrace{\Delta p(a) \chi \eta(t)}_{\text {noise }}$

where the subscript ' $d$ ' denotes 'data', $\Delta p(a)=p_{s s}(a)-p_{0}$, is the difference between SS and IC for DEP collection, $\chi$ is an attenuation constant and $\eta(t)=N(0,1)$ is a Gaussian (normal) random variable with zero mean and unit variance. The attenuation was set to $5 \%$ which corresponds the range of noise typically observed in reports by us $[16,17]$ so that it mimics experimental measurements.

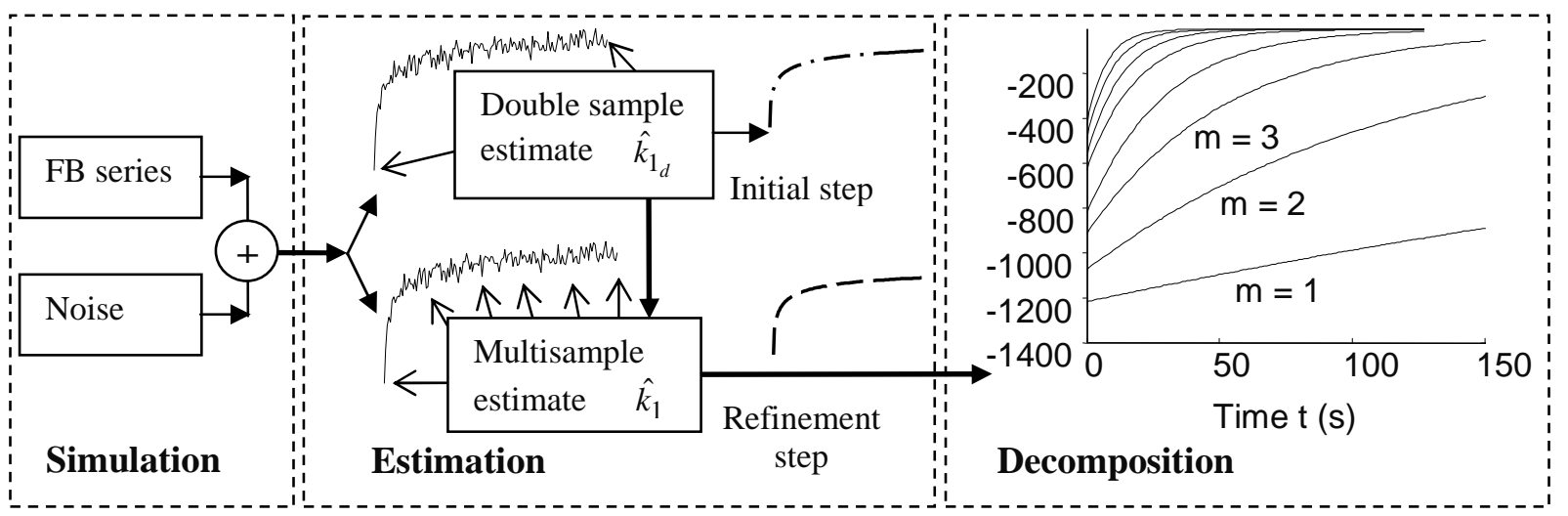

Figure 9. Schematic of simulation, two-step estimation and decomposition.

Parameter values used to simulate data were $a=0 \mu \mathrm{m}, w+g=20 \mu \mathrm{m}, h=100 \mu \mathrm{m}, k_{l}=1 \mathrm{fN}$. The initial estimation step used two time points, or samples, corresponding to initial and quasi-SS, as shown in figure 10. The algorithm was the inverse, nonlinear solution to the SS pdf (3.1) and the two sample estimate was evaluated using the Newton-Raphson root finding method that solved with a nominal starting value, $k_{1}=0$. The details are given in Appendix C; the initial estimate, corresponding to figure 10 was $\hat{k}_{l_{d}}=0.945 \mathrm{fN}$ where sub-subscript ' $\mathrm{d}$ ' denotes the initial double sample estimate. The second refinement step used the initial estimate $\hat{k}_{1_{d}}$ and other parameter values as before. The FB series generated the time profile and the difference between noisy data was minimised using method of Least Squares Error (LSE). LSE minimisation was performed using Nelder-Mead (NM) optimisation in Matlab ${ }^{\mathrm{TM}} 7.8$ with the Sum of the Squares of Errors (SSE) criteria for the $i$ th iteration,

$S S E_{i}=\sum_{s=1}^{s_{x}}\left[p_{d}\left(a, t_{s}\right)-p\left(\hat{k}_{1}^{i}, a, t_{s}\right)\right]^{2}$ 
where $s_{x}$ is the maximum integer for the multiple point sample index, $s$. In (4.8) $t_{s}$ denotes the discrete time point and it is understood $\hat{k}_{1}^{i}$ is the $i$ th iterative estimate of $k_{1}$. Minimising the SSE yielded estimates that were close to the true (simulation without noise) value. The multi-sample estimate, $s_{x}=150$ for the noisy data example depicted in figure 10 was $\hat{k}_{1}=0.998 \mathrm{fN}$. The profile generated by the FB series using the multisample estimate practically overlays the true profile (generated using $k_{l}$ ). The reason for underestimation in the double sample case is due to the time profile not completely reaching SS at 150 s, i.e. the value taken from the time profile at $150 \mathrm{~s}$ is less than the actual SS, $p(a, 150)<p_{s s}(a)$. A colour view and higher magnification showing the plots is available at the publisher's website.

The utility of the two-step estimation method was confirmed by repeating the estimation procedure a hundred times, i.e. Monte Carlo (MC) simulations. The NM optimisation routine required multiple evaluations of the FB series so the linear approximation (4.6) was used to hasten multi-sample estimation and LSE evaluation. Despite the limitations of the approximation in terms of accuracy, the advantages of multi-sample estimation, which incurred little extra computation time, is shown by the histograms of the $k_{l}$ estimates in figure 11. The histogram of $k_{l}$ using MC exhibits a mean, $\mu\left(\hat{k}_{1}\right)=0.98$ and standard deviation (s.t.d), $\sigma\left(\hat{k}_{1}\right)=0.021 \mathrm{fN}$, is narrow and close to the true value, $k_{1}=1 \mathrm{fN}$. On the other hand, the histogram of double sample estimates is broader and the mean, $\mu\left(\hat{k}_{1_{d}}\right)=0.91 \mathrm{fN}$, is further away from the true value and s.t.d wider, $\sigma\left(\hat{k}_{1_{d}}\right)=0.081$. These trends are in accordance with the central limit theorem where smaller samples lead to estimates that will vary about the true value [35]. The results demonstrate that the DEP force can be estimated, $\hat{k}_{1}=1 \mathrm{fN}$, that is very close to the true value solely from the experimental data and without needing an informed starting guess. The results also show that temporal information can be used by the FB series to refine the initial estimate from SS data.

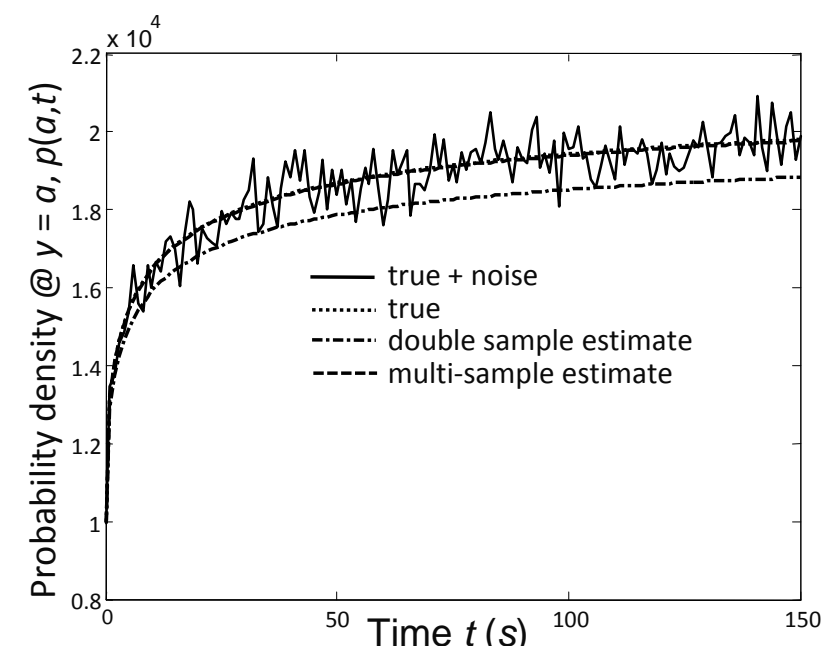

Figure 10. Estimation of DEP force coefficient, $k_{l}$ from simulated noisy data by two methods. The multiple sample estimate is more effective than the two point estimator so that the time profile is very close to the true value $k_{l}=1 \mathrm{fN}$. See text for details.

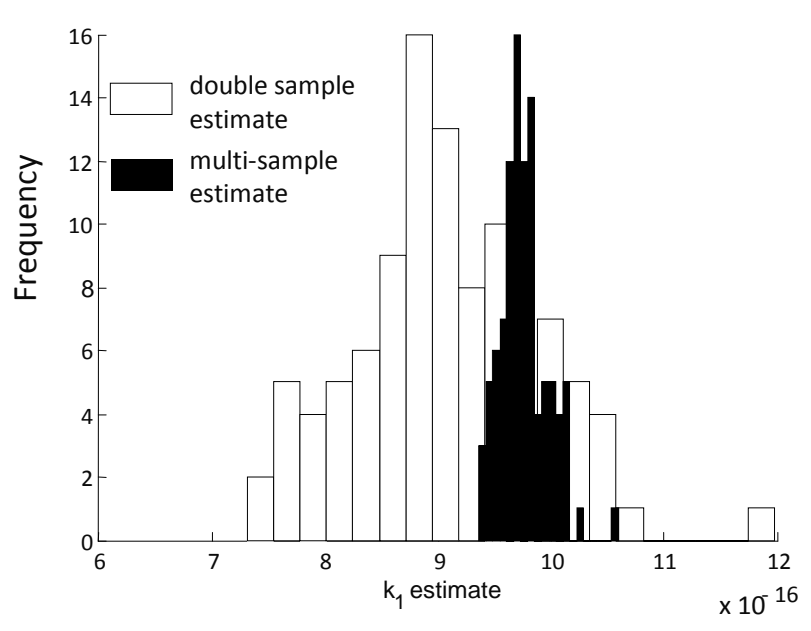

Figure 11. Estimation of DEP force coefficient, $k_{l}$. Comparison of the two histograms of estimates shows that the multiple sample estimate is a more efficient estimator with smaller standard deviation. Its mean is also closer to the true value $k_{l}=1 \mathrm{fN}$.

Accomplishing a best-fit time profile by using the multi-sample estimate, $\hat{k}_{1}=1 \mathrm{fN}$, immediately allows eigenmode decomposition, as shown, in figure 12. All spatial eigenmode terms are shown to be negative for this case $y=0$ and clearly shows that the first $(m=1)$ term being the greatest in magnitude, followed by the second, third, and so forth. This ordering follows as the DEP force is varied - data not shown, particularly for 
the transition from weak to strong DEP. This means that the series can be safely truncated and that there is negligible loss of information.

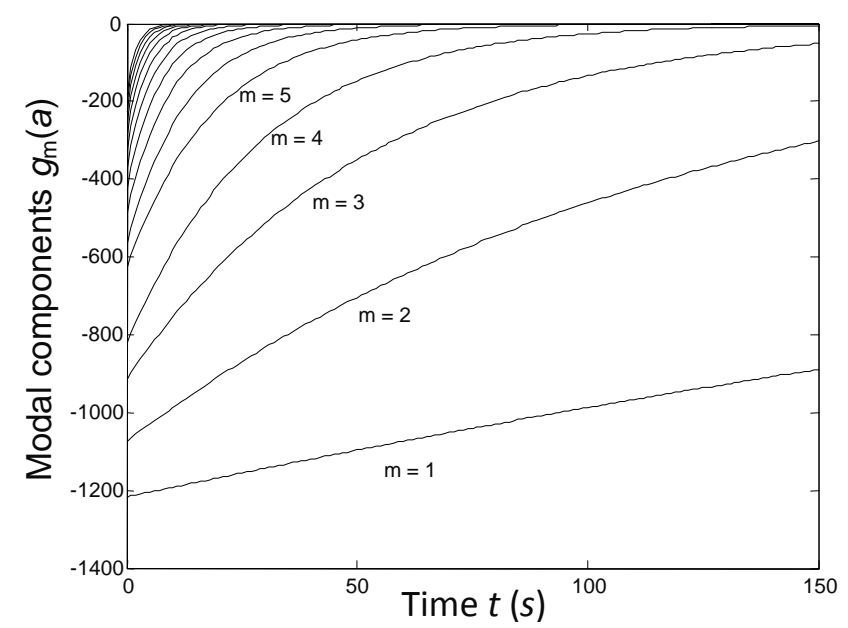

Figure 12. Principle of decomposition applied to pseudoexperimental data showing that the first eigenmode dominates DEP-driven nanoparticle collection, followed by the second, etc.

\subsubsection{Single eigenmode decomposition}

One of the features of the time profile fitting method is that that it inherently combines all the eigenmode terms together, uses all the temporal data, and only the spatial data at a single position, $y$. In an envisaged operational system not all of the data may be available so a complementary approach would be useful. An instructive alternative is to select one of eigenmode terms, use data from a single time instant, and all of the spatial data. A conceptual schematic of the spatial data decomposition process is shown in figure 13. Experimental concentration data, $c(y, t)$ from a DEP collection experiment at a time instant, $t=t_{i}$ is normalised using (2.2) to obtain the density, $p\left(y, t_{i}\right)$. It is then filtered to retain the mode of interest, $m=n$, and remove all the other unwanted eigenmodes, $m \neq n$. This results in a numerical value corresponding to the strength of the selected mode.

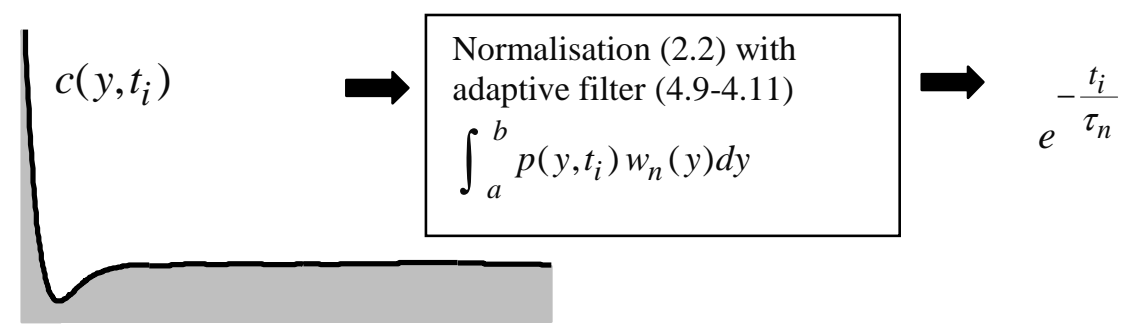

Figure 13. Adaptive filter uses spatial nanoparticle collection information (figure 6 cross-section of surface at time $t_{i}=5 \mathrm{~s}$ ) to extract selected eigenmode $n$.

To achieve filtering a particular mode, a suitable spatial function, $w_{n}(y)$, is devised that weights the $p\left(y, t_{i}\right)$ in such a way that their product integrated over the entire chamber height selects a single eigenmode $n$, and automatically excludes the other terms in the series. The spatial variable, $y$, is eliminated by integration. Hence, at a particular time, $t=t_{i}$, an integral operator $I_{n}$ achieves a theoretical value, 
$I_{n}\left(t_{i}\right)=\int_{a}^{b} p\left(y, t_{i}\right) w_{n}(y) d y=\delta_{m n} e^{-\frac{t_{i}}{\tau_{n}}}$

In (4.9) the Kronecker delta function signifies unity for the $m=n$th mode and the remaining, $m \neq n$ are zero. The modal index, $m$ arises from the probability density given by (3.13). Recalling the orthogonal basis established in Appendix A, suggests a suitable normalised Bessel weight function that is capable of selecting the $n$th eigenmode as,

$w_{n}(y)=\frac{e^{c(y)} \tilde{g}_{n}(y) e^{-\lambda e^{-k_{2} y}}}{\int_{a}^{b} e^{c(y)} \tilde{g}_{n}(y) e^{-\lambda e^{-k_{2} y}} d y}$

Algebraic simplifications, for example, for the SS eigenmode, $n=0$, show that $w_{0}(y)=1$ thus concurring with the axiomatic condition (2.2). Numerical evaluation of the weight function requires an initial estimate of $k_{1}$, in addition to the others (e.g. $k_{2}$ ) so that the filter is adaptive. Once the initial parameters are assigned, the time exponential in (4.9) can provide an iterative method for estimating $k_{1}$ from experimental data $c(y, t)$ with unknown parameter values. An error criteria for iterative feedback for estimating $k_{1}$, or as a evaluation performance measure, is

$\varepsilon_{n}=\left|I_{n}\left(t_{i}\right) / e^{-t_{i} / \tau_{n}}-1\right|$

Ideally, the cross-sectional spatial information is inputted into the filtering operation given by (4.10) and (4.11) and yields a real number equalling the temporal value, as shown in figure 13. However, there is some numerical error arising from the numerical integrations performed in Matlab ${ }^{\mathrm{TM}} 8$. Decompositions of simulated data $p(y, t)$ using (4.9-4.11) have shown the relative error of the numerically evaluated integral, $I_{n}\left(t_{i}\right)$, compared with the theoretical value $e^{-t_{i} / \tau_{n}}$ concurs to within less than $1 \%$ for the first three eigenvalues $(n=1, . ., 3)$. To illustrate the error with an example, reference is made to figure 13 where the plot on the left hand side is the cross-section of the surface, $p(y, t)$ illustrated in figure 6 at $t_{i}=5 \mathrm{~s}$. Recall that the simulated nanoparticle collection used $k_{1}=1 \mathrm{fN}$ and other parameters as previously stated, and a simple normalization implies $N=1$. Selecting the second eigenmode, $n=2$, for example, with eigenvalue, $\rho_{2}=0.091876$ or mode time constant $\tau_{2}=118.47 \mathrm{~s}$ resulted in a numerical value for the left hand integral in (4.9), $I_{2}(5)=0.95447$ and was evaluated using adaptive Lobatto quadrature in Matlab ${ }^{\mathrm{TM}}$ 7.8. The right hand side of (4.9) yields a theoretical value, $e^{-5 / 118.47}=0.95867$ hence, from (4.11), $\varepsilon=4.4 \times 10^{-3}$. Other numerical integrations show that the remaining $m \neq n$ modes were extremely low compared with the selected mode, typically seven orders of magnitude higher. This means that not only is there capability for decomposing inputted data in time (along the time axis) at a selected point in space, as shown in (4.3.1), but capability for decomposing data in space (along the positional y axis) at a instant in time.

\subsection{Domain evaluation and representation}

The solution demonstrates that a systematic analysis of DEP nanoparticle transport combined with diffusion results in a series of exponential time responses with Bessel function amplitudes predicting the DEP force strength associated with each mode. Nanoparticle collection and release in a DEP microdevice shown in figure 2 has a temporal response predicted by the exponential time decay terms in (3.13) and (3.14). Since DEP as an electrokinetic transport driver shows considerable promise the time response will be important for 
monitoring, control and other purposes. It is foreseeable that representation in alternative domains could be an important tool for the development of these microdevices so an example is given for the frequency domain.

To find the frequency response, the time derivative of the solution (3.11) is evaluated and the expression is Fourier transformed [36]. Since the distributional derivative of the step input is an impulse function, the output can be said to be an 'impulse response' provided certain assumptions are kept in mind as discussed later. The derivative of the series (3.12) is,

$$
\dot{p}(y, t)=\left[p_{s}(y)+\sum_{m=1}^{\infty} C_{m} e^{c(y)} \tilde{g}_{m}(y)\right] \delta(t)-\sum_{m=1}^{\infty} \frac{1}{\tau_{m}} C_{m} e^{c(y)} \tilde{g}_{m}(y) \mathrm{e}^{-\frac{t}{\tau_{m}}} u(t)
$$

where the dot, ".' denotes the time derivative and $\delta(t)$ is the Dirac delta function. Our interest lies in the sum of exponential decay terms shown in the second term in (4.12). Both (3.12) and (4.12) are causal, hence the Fourier transform is

$$
P_{c}(y, f)=F\{\dot{p}(y, t)\}=\int_{-\infty}^{\infty} \dot{p}(y, t) e^{-i \omega t} d t=-\sum_{m=1}^{m_{c}} g_{m}(y) H_{m}(f)
$$

where the angular frequency is $\omega=2 \pi f$ and $H(f)=\frac{1}{1+i \omega \tau_{m}}$ is the Fourier transform of the derivative of the $m$ th exponential decay term in the series. As for the time domain for the collection process, the infinite series is evaluated by truncating to $m_{c}$ and is a trade-off between computation time, accuracy needed for the application and convergent properties of the series. Similarly, the frequency response of a single eigenmode can be evaluated, for example, by Fourier transforming the Bessel filter given by (4.9). Combining both weighting and transform operations, the integral is Fourier-Bessel (FB) operator with subscript ' $n$ ' signifying the $n$th mode

$$
F B_{n}\{p(y, t)\}=\int_{-\infty}^{\infty} \int_{a}^{b} p(y, t) w_{n}(y) e^{-i 2 \pi f t} d y d t=\frac{-1}{1+i \omega \tau_{n}}
$$

The frequency spectrum of DEP time dependent transport is rapidly evaluated and is shown in figure 14. The parameter values are for the perturbation case, $k_{l}=1 \mathrm{fN}, a=0 \mu \mathrm{m}$, electrode dimensions $w+g=20$ $\mu \mathrm{m}, h=100 \mu \mathrm{m}$ with the spatial point of interest set at the lower boundary, $y=a$. The series (4.13) converged less rapidly that the time domain form (3.13) but sufficient accuracy was obtained using $m_{c}=40$. The plot for the multiple eigenmode sum given by (4.13) exhibits rounded roll-off due compared with a single, first eigenmode term in the series, also plotted in figure 14. It illustrates the very slow time response of the first, main, eigenmode, with $\tau_{1} 478 \mathrm{~s}$, that is also apparent in the time domain decomposition, shown in figure 12 .

The ultra-low frequency roll-off arises from the diffusion over the $100 \mu \mathrm{m}$ chamber height that acts to dampen high frequencies arising from the DEP step-force. Note that a normalised version of the first eigenmode term plotted in figure 14, without the spatial term $g_{1}(a)$, could have been evaluated from (4.14) rather than (4.13). It is important to be careful interpreting the frequency spectrum plotted in figure 14; it is not expected to be the same as the classical impulse response associated with linear time invariant (LTI) systems [36]. The reason (3.13) is not LTI is because the nanoparticle density is spatially distributed, as shown by the propagating depletion zone in figure 6 . That is, the assumption of a uniform spatial distribution of nanoparticles for the IC used to evaluate (3.13) is not entirely valid, rather it is an approximation. However, under weak DEP forces that merely perturb the system, is expected to be quasi-LTI so the frequency response is not exact but a useful indication of the DEP driven nanoparticle transport in a microdevice. 


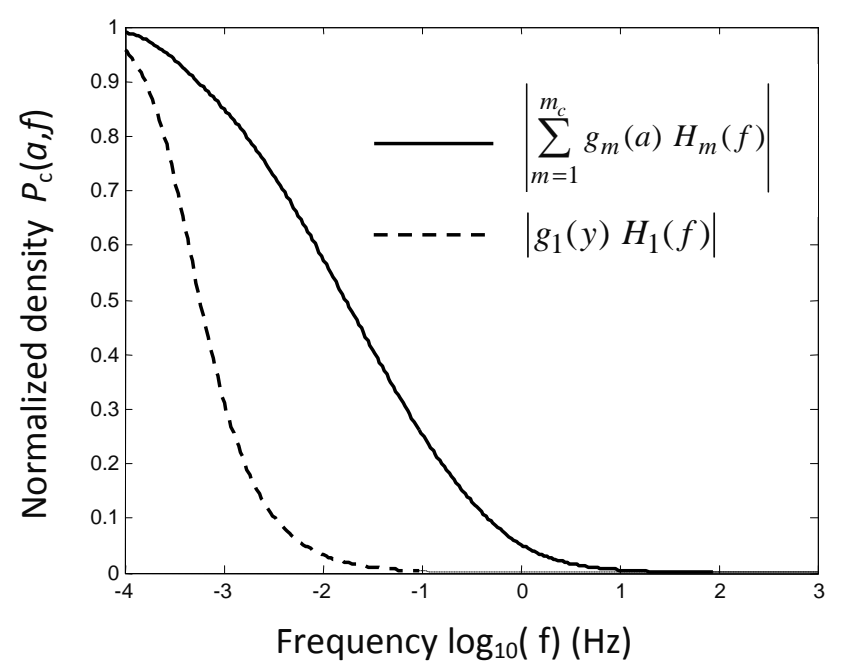

Figure 14. Frequency response of DEP collection for step DEP input evaluated at $y=a$ showing differences between the multiple eigenmode and single eigenmode $(n=1)$ with a less rounded cut-off.

Ultra-low time modulated DEP causes nanoparticles to be cyclically drawn to, and released from, the electrode surface. As the modulation frequency is increased, however, there occurs a frequency when the nanoparticles barely move. No sooner are the nanoparticles attracted to the electrode surface by DEP, they are released into the bulk under the action of diffusion as the force decreases in the cycle. The frequency response in figure 14 indicates that if the DEP force is modulated in time with frequency of the order of tens of Hertz or higher, there would be little modulation of nanoparticles observed - apart from the time averaged DC level that results in a partial SS 'on' response. The time-averaged DC level arises from the fact that the DEP force is proportional to the square of the applied voltage and the phenomenon is identifiable by laboratory modulation experiments. Under these caveats, it is possible to suggest a new parameter in investigations as the 'DEP modulation bandwidth', although the topic strictly awaits a more detailed investigation. This includes a definition for the cut-off frequency, $f_{c}$, that should be capable of being observed experimentally. If a constant $\left\{k_{c}: 0<k_{c}<1\right\}$ is assigned as the proportion of the maximum, then

$$
f_{c}:\left|P_{c}(y, f)\right|=k_{c}\left|P_{c}(y, f)\right|_{\max }
$$

For example, $k_{c}=1 / \sqrt{2}$ is defined as the half-power frequency for resistor-capacitor LPF circuits [36]. Due to a rounded roll-off and that a sensible laboratory criterion is that 'almost all' of the nanoparticles are not being modulated, then $k_{c}=0.05$. Applying this criterion to the figure 14 yields about $f_{c}=7$ milliHertz (mHz) for the first eigenmode and for the entire density $f_{c}=1 \mathrm{~Hz}$. Laboratory DEP modulation experiments using nanospheres with the same $216 \mathrm{~nm}$ diameter as the simulation indicate this value of the order of $1 \mathrm{~Hz}$ is reasonable and, hence, supports the predictions by the FB model.

\subsection{Representation}

Each of the modal frequency terms in (4.13) is identifiable with a Low-Pass Filter (LPF) equivalent circuit so that the expression for the sum is represented by the schematic shown in figure 15. In the schematic, each of the active-LPFs (A-LPFs) entails a resistor-capacitor filter with values that govern each eigenmode time constant, $\tau_{m}$, and an active operational amplifier (op-amp) that compensates any resistive losses thus 
representing each spatial $g_{m}(y)$ term. The Fourier transform of the nanoparticle release process can also be represented by a similar frequency domain approach. For weak DEP with $k_{l}=0.1 \mathrm{fN}$ at the lower boundary at $y=a$ it was shown that the time profiles are complementary, as given by (4.2). Hence, in this case, the absolute value of the frequency response of the release should be the same as the collection and the schematic representation will include a sum of A-LPFs similar to figure 15.

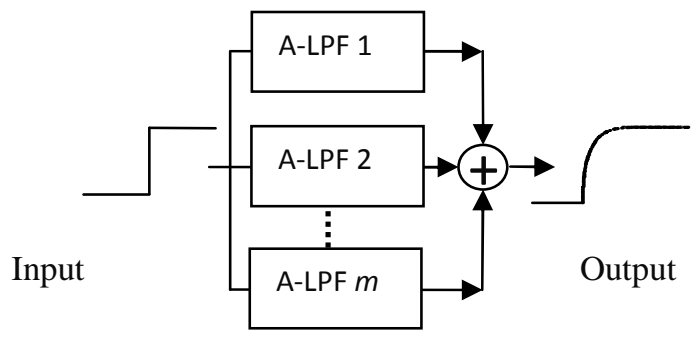

Figure 15. Nanoparticle DEP collection process for a DEP step input at $y=a$ can be considered as sum of active low pass filters, each with its own gain and cut-off frequency. For low DEP forces, the representation is applicable for nanoparticle release.

\section{Discussion and concluding remarks}

A Fourier-Bessel series solution to a 1D linear FPE, or modified diffusion, equation has been developed to predict nanoparticle concentration as a function of position and time. The model is applicable for noninteracting nanoparticles transported by a spatial DEP force that varies exponentially in a microdevice, as arises from a planar interdigitated array. The model allows DEP to be switched on then off in time and classifies the collection and release dynamics of nanoparticle transport as a microdevice system perturbation for weak DEP force coefficients up to $1 \mathrm{fN}$. This is responsible for small proportions of nanoparticles collecting under the action of positive DEP near the array and being released when the force is switched off. The perturbation classification contrasts with state transition where much stronger DEP forces significantly concentrate nanoparticles in regions close to the array, and depletion occurs elsewhere in the microdevice chamber. The classifications in sections 3.1 and 4.1, based on both SS and time responses, are as expected for a system that combines DEP and thermal diffusion.

Assuming weak DEP forces where the nanoparticle concentration is perturbed, the FB model was used to develop and demonstrate four other key applications areas: approximation, estimation and decomposition, transformation and representation. There is considerable opportunity for further work. The approximations that saved computation time, particularly for the estimation and decomposition application, could be generalised to include other positions above the array. Of interest are the eigenmode time constants in figure 5 that were shown to remain relatively constant so that the nanoparticle collection time profile at the array could be rescaled for small increases in the DEP force. The two-stage estimation process for the DEP time response that successfully estimated the DEP force coefficient could be developed further for other parameters, e.g. the electrode dimension coefficient, $k_{2}$. Although $k_{2}$ was assigned as 'known', our interest also lies the inferring its value from experimental data, i.e. its effective value. Clearly, the single eigenmode adaptive filter that used of all spatial information and only one time point for a chosen eigenmode, could be developed further for robustness against noise that typifies quantitative laboratory measurements using fluorescence microscopy. The A-LPF equivalent representation could also be investigated for positions away from the array, e.g. at $20 \mu \mathrm{m}$, that become temporarily depleted of nanoparticles, as shown in figure 6 . Preliminary results suggest nanoparticle depletion could be represented by adding circuit elements that introduce $180^{\circ}$ phase shift, such as inductors, to the resistor-capacitor A-LPFs or by inverting the gain of the 
op-amps. As with many of the applications, there is scope also for representation that considers high DEP force collection of nanoparticles followed by their release into the bulk solution.

The time response of the first decomposed and dominant eigenmode of about eight minutes for a typical DEP collection experiment indicates a possible benchmark for future experiments involving ultra-low frequency time modulated DEP. Currently, sinusoidal and square wave 'on/off' switched DEP is being investigated in our laboratory and suggests a low frequency DEP modulation bandwidth of the order of one Hertz and this concurs with the FB series that predicts a nanoparticle transport process damped by diffusion. This is a preliminary finding and the prediction is for a 1D system whereas experiments occur in 3D microchamber. This motivates further development of a 3D software tool for analysing quantitatively measured time data of DEP nanoparticle collection experiments and the separation of variables method can be readily extended to 2D and 3D. All numerical evaluations were performed in Matlab ${ }^{\mathrm{TM}} 7.8$ and were verified by independent FEM simulations using FlexPDE ${ }^{\mathrm{TM}}$ 2.15. Computing environments, such as, Matlab ${ }^{\mathrm{TM}} 7.8$, that are based on matrix calculus are suited to evaluating series. In general, the FB series took longer to compute than FlexPDE ${ }^{\mathrm{TM}}$ 2.15. This was attributed to the high accuracy needed for computing the integration constants, particularly the eigenvalues that involved root evaluation and spatial integration that used quadrature methods. However, once the eigenvalues and FB spatial terms had been evaluated for a set of parameter values, re-evaluation on different time scales was very fast compared with FlexPDE ${ }^{\mathrm{TM}} 2.15$ that needed to be re-run. Importantly, evaluation in alternative domains, such as, the frequency domain was easily achieved with the FB model and computation time could be reduced by coding mathematical algorithms in other languages, such as, $\mathrm{C}^{++}$or Fortran 90. Applications are anticipated to be integrated and developed into an information software tool with a user-friendly interface, e.g. Matlab ${ }^{\mathrm{TM}} 7.8$, and to be made freely available.

The development of applications, particularly that involve time responses, is important for the development of DEP-based LOC and novel technologies, such as, scanning probe microscopes, quantum dots and optical devices $[5-8,10,12,13]$. Fourier transformations of experimental data, for example, are routine in today's electronics industry for frequency domain analysis and testing, and Laplace and z-transforms are used for automatic control stability analysis and design. Further work also includes the development of a nonlinear FPE that could take into account the effect the nanoparticles have on the electric field and influence of other electrokinetic effects, such as, electro-osmosis. In this respect the linear FB model will provide an important framework for analysis and comparison. Applications of the Fourier-Bessel series for modelling nanoparticle transport appear to be the first of its kind in the literature on DEP and engenders a microdevice systems approach. It shows considerable promise as a development tool for understanding and advancing DEP applications for transporting nanoparticles.

\section{ACKNOWLEDGEMENTS}

The author thanks Drs. Jason Ralph and Judy Zhu in the Department of Electrical Engineering and Electronics, University of Liverpool, for helpful comments. 


\section{Appendix A}

The third set of integration constants is evaluated by finding an orthogonal basis. A suitable weighting function is defined

$q_{n}(y)=g_{n}(y) / p_{s s}(y)=\frac{g_{n}(y)}{p_{c} \exp \left(\lambda e^{-k_{2} y}\right)}, \lambda \neq 0$

where $p_{s}(y)$ is the SS solution given by (3.1), $p_{c}=\frac{k_{2}}{E i\left(\lambda e^{-k_{2} a}\right)-E i\left(\lambda e^{-k_{2} b}\right)}$ and the general case, $\lambda \neq 0$ is given. In (A.1) $g_{n}(y)$ is a spatial eigenmode term given in (3.6) and $n$ is an eigenmode index. Setting $t=0$ in the series pdf (3.2) removes time dependence. Multiplying the right hand side (rhs) of (3.2) by weight (A.1) and integrating over the interval from $y=a$ to $y=b$

$\int_{a}^{b} p(y, 0) q_{n}(y) d y=\sum_{m=0}^{\infty} \int_{a}^{b} q_{n}(y) g_{m}(y) d y$

Summing the left hand side (lhs) of (A.2) over the $n$ indices,

$$
\begin{aligned}
\sum_{n=0}^{\infty} \int_{a}^{b} p(y, 0) q_{n}(y) d y & =\sum_{n=0}^{\infty} \sum_{m=0}^{\infty} \int_{a}^{b} g_{m}(y) q_{n}(y) d y \\
& =\sum_{n=m=0}^{\infty} \int_{a}^{b} g_{m}(y) q_{n}(y) d y+\sum_{n \neq m}^{\infty} \int_{a}^{b} g_{m}(y) q_{n}(y) d y
\end{aligned}
$$

By definition an orthogonal function has properties,

$$
\int_{a}^{b} g_{m}(y) q_{n}(y) d y=c_{c} \delta_{m n}=\left\{\begin{array}{l}
c_{n}, m=n \\
0, m \neq n
\end{array}\right.
$$

where $c_{n}$ is a normalisation constant and $\delta_{m n}$ is the Kronecker delta function.

Applying (A.4) to (A.3) and substituting (A.1)

$$
\sum_{m=0}^{\infty} \int_{a}^{b} p(y, 0) \frac{g_{m}(y)}{p_{c} \exp \left(\lambda e^{-k_{2} y}\right)} d y=\sum_{m=0}^{\infty} \int_{a}^{b} g_{m}(y) \frac{g_{m}(y)}{p_{c} \exp \left(\lambda e^{-k_{2} y}\right)} d y
$$

From (3.8) $g_{m}(y)=C_{m} e^{c(y)} \tilde{g}_{m}(y)$ hence each $l h s$ and $r h s$ term in (A.5) becomes,

$$
\frac{C_{m}}{p_{c}} \int_{a}^{b} p(y, 0) e^{c(y)} \tilde{g}_{m}(y) e^{-\lambda e^{-k_{2} y}} d y=\frac{C_{m}{ }^{2}}{p_{c}} \int_{a}^{b} e^{2 c(y)} \tilde{g}_{m}{ }^{2}(y) e^{-\lambda e^{-k_{2} y}} d y
$$

and (3.10) follows. 


\section{Appendix B}

The $m=0$ term of the series is verified by setting $\rho_{0}=0$ so that from (3.3)

$$
h_{0}(t)=1
$$

and from expressions in (3.5), $\alpha=\frac{1}{2}, I^{\dagger}\left(\frac{1}{2}, \kappa(y)\right)=\sqrt{\frac{2}{\pi \kappa(y)}} e^{\kappa(y)}$ and $K^{\dagger}\left(\frac{1}{2}, \kappa(y)\right)=0$. Hence, from (3.6)

$$
g_{0}(y)=C_{0} e^{c(y)} \tilde{g}_{0}(y)=C_{0} e^{c(y)} \sqrt{\frac{2}{\pi \kappa(y)}} e^{\kappa(y)}=C_{0} e^{\lambda e^{-k_{2} y}} \frac{2}{\sqrt{\pi \lambda}}
$$

and $\lambda$ is defined in (3.1). The numerator of the integration constant $C_{0}$ defined in (3.10) is

$$
C_{0_{\text {num }}}=\int_{a}^{b} p(y, 0) e^{c(y)} \tilde{g}_{0}(y) e^{-\lambda e^{-k_{2} y}} d y=\frac{2}{\sqrt{\pi \lambda}} \int_{a}^{b} p(y, 0) d y=\frac{2}{\sqrt{\pi \lambda}}
$$

Similarly the denominator of the integration constant $C_{0}$ is

$$
C_{0_{d e n}}=\int_{a}^{b} e^{2 c(y)} \tilde{g}_{m}(y)^{2} e^{-\lambda e^{-k_{2} y}} d y=\frac{4}{\pi \lambda} \int_{a}^{b} e^{\lambda e^{-k_{2} y}} d y=\frac{4\left[E i\left(\lambda e^{-k_{2} a}\right)-E i\left(\lambda e^{-k_{2} b}\right)\right]}{\pi \lambda k_{2}}
$$

where the exponential integral identity is found by change of variable. Thus,

$$
C_{0}=\frac{C_{0_{\text {num }}}}{C_{0_{\text {den }}}}=\frac{k_{2} \sqrt{\pi \lambda}}{2\left[E i\left(\lambda e^{-k_{2} a}\right)-E i\left(\lambda e^{-k_{2} b}\right)\right]}
$$

Substituting (B.5) into (B.2) and using the relations in (3.5)

$$
g_{0}(y)=\frac{k_{2} e^{\lambda e^{-k_{2} y}}}{\left[E i\left(\lambda e^{-k_{2} a}\right)-E i\left(\lambda e^{-k_{2} b}\right)\right]} \equiv p_{c} e^{\lambda e^{-k_{2} y}}
$$

Combining (B.1) and (B.5), the first term is

$$
g_{0}(y) h_{0}(t)=p_{c} e^{\lambda e^{-k_{2} y}} \equiv p_{s s}(y)
$$

which is the relation for the collection SS in (3.1) and (3.12) follows. Note that the IC in the integrals of B.3 are arbitrary. Also, it can be shown that in the case when the DEP force is switched is off, the limiting values $k_{1} \rightarrow 0$ and $\lambda \rightarrow 0$, enables the denominator in (B.6) to simplify

$$
E i\left(\lambda e^{-k_{2} a}\right)-\left.E i\left(\lambda e^{-k_{2} b}\right)\right|_{\lambda \rightarrow 0}=k_{2}(b-a)
$$

using series expansions for the $E i$ functions [34]. Hence the above argument applies for nanoparticle release and (3.12) follows. 


\section{Appendix C}

The value for the DEP force coefficient $k_{1}$ is found from initial and final experimental measured concentrations of collection profiles and evaluated using the relative amplitude parameter, $A(\mathrm{y})$, defined in (2.3). Inverting (2.3) and (3.1) yields $k_{l}$. Since (3.1) is nonlinear, $k_{l}$ is found iteratively by establishing a general function depending on $k_{l}$,

$$
f\left(k_{1}\right)=k_{1}-k_{2} k_{B} T e^{k_{2} y}\left[\ln (A+1)+\ln \left\{\operatorname{Ei}\left(\frac{k_{1}}{k_{2} k_{B} T} e^{-k_{2} a}\right)-\operatorname{Ei}\left(\frac{k_{1}}{k_{2} k_{B} T} e^{-k_{2} b}\right\}-\ln \left\{k_{2}(b-a)\right\}\right]\right.
$$

Let the superscript, ' $i$ ', denote the $i$ th iteration. The root, $f\left(k_{1}\right)=0$, is found using Newton's method [34],

$k_{1}^{i+1}=k_{1}^{i}-\frac{f\left(k_{1}^{i}\right)}{f^{\prime}\left(k_{1}^{i}\right)}$

where ' denotes derivative with respect to $k_{l}$. For convenience, let

$\Delta E i\left(k_{1}\right)=E i\left(\frac{k_{1}}{k_{2} k_{B} T} e^{-k_{2} a}\right)-E i\left(\frac{k_{1}}{k_{2} k_{B} T} e^{-k_{2} b}\right)$

Hence, differentiating (C.1) using the chain rule,

$\frac{d f}{d k_{1}}=1-k_{2} k_{B} T e^{k_{2} y} \frac{d \Delta E i / d k_{1}}{\Delta E i\left(k_{1}\right)}$

The exponential integral function given by the CPV properties yields [34], $\frac{d E i}{d k_{1}}=\frac{e^{\lambda e^{-k_{2} y}}}{k_{1}}$ so

$\frac{d \Delta E i}{d k_{1}}=\frac{e^{\lambda e^{-k_{2} a}}-e^{\lambda e^{-k_{2} b}}}{k_{1}}$

Combining (C.1), (C.4), (C.5) and (C.2) enables a good approximation of the root and hence estimate for $k_{l}$. Noting that the series expansions for the Ei and exponential functions [34]

$$
\left.\Delta E i\left(k_{1}\right)\right|_{k_{1} \rightarrow 0}=k_{2}(b-a)
$$

and

$\left.\frac{d \Delta E i}{d k_{1}}\right|_{k_{1} \rightarrow 0}=\frac{e^{-k_{2} a}-e^{-k_{2} b}}{k_{2} k_{B} T}$

enables use of a default starting value, $k_{1}=0$, for the starting, $i=0^{\text {th }}$, iteration. 


\section{REFERENCES}

1. Reyes, D.R., et al., Micro total analysis systems. 1. Introduction, theory, and technology. Analytical Chemistry, 2002. 74(12): p. 2623-2636.

2. Lab on a Chip. [cited; Available from: http://pubs.rsc.org/en/Journals/JournalIssues/LC.

3. Abidin, Z.Z., L. Downes, and G.H. Markx, Novel electrode structures for large scale dielectrophoretic separations based on textile technology. Journal of Biotechnology, 2007. 130(2): p. 183-187.

4. Abidin, Z.Z., L. Downes, and G.H. Markx, Large scale dielectrophoretic construction of biofilms using textile technology. Biotechnology and Bioengineering, 2007. 96(6): p. 1222-1225.

5. Hilton, A.M., B.P. Lynch, and G.J. Simpson, Reduction of tip-sample contact using dielectrophoretic force scanning probe microscopy. Analytical Chemistry, 2005. 77(24): p. 8008-8012.

6. $\quad$ Lynch, B.P., et al., Dielectrophoretic force microscopy of aqueous interfaces. Langmuir, 2005. 21(4): p. 14361440 .

7. Gallo-Villanueva, R.C., et al., DNA manipulation by means of insulator-based dielectrophoresis employing direct current electric fields. Electrophoresis, 2009. 30(24): p. 4195-4205.

8. Lapizco-Encinas, B.H., S. Ozuna-Chacon, and M. Rito-Palomares, Protein manipulation with insulator-based dielectrophoresis and direct current electric fields. Journal of Chromatography A, 2008. 1206(1): p. 45-51.

9. Yunus, N.A.M. and N.G. Green, Continuous Separation of Submicron Particles Using Angled Electrodes - art. no. 012068, in Electrostatics 2007, N. Green, Editor. 2009, Iop Publishing Ltd: Bristol. p. 12068-12068.

10. Hakala, T.K., et al., Field-Induced Nanolithography for High-Throughput Pattern Transfer. Small, 2009. 5(23): p. 2683-2686.

11. Cheon, D., S. Kumar, and G.H. Kim, Assembly of gold nanoparticles of different diameters between nanogap electrodes. Applied Physics Letters. 96(1).

12. Sin, M.L.Y., et al., Active Manipulation of Quantum Dots using AC Electrokinetics. Journal of Physical Chemistry C, 2009. 113(16): p. 6561-6565.

13. Kayani, A.A., et al., Novel tuneable optical elements based on nanoparticle suspensions in microfluidics. Electrophoresis. 31(6): p. 1071-1079.

14. Pohl, H.A., Dielectrophoresis. 1978, Cambridge: Cambridge University Press.

15. Bakewell, D.J. and H. Morgan, Measuring the frequency dependent polarizability of colloidal particles from dielectrophoretic collection data. IEEE Transactions on Dielectrics and Electrical Insulation, 2001. 8(3): p. 566571.

16. Bakewell, D.J. and H. Morgan, Dielectrophoresis of DNA: Time- and frequency-dependent collections on microelectrodes (vol 5, pg 1, 2006). IEEE Transactions on Nanobioscience, 2006. 5(2): p. 139-146.

17. Bakewell, D.J. and H. Morgan, Quantifying dielectrophoretic collections of sub-micron particles on microelectrodes. Measurement Science \& Technology, 2004. 15(1): p. 254-266.

18. Gunda, N.S.K. and S.K. Mitra, Modeling of dielectrophoretic transport of myoglobin molecules in microchannels. Biomicrofluidics. 4(1): p. Article No.: 014105.

19. Sun, T., H. Morgan, and N.G. Green, Analytical solutions of ac electrokinetics in interdigitated electrode arrays. Physical Review E, 2007. 76: p. 046610.

20. Morgan, H., et al., The dielectrophoretic and travelling wave forces generated by interdigitated electrode arrays: analytical solution using Fourier series. Journal of Physics D-Applied Physics, 2001. 34(10): p. 15531561.

21. Price, J.A.R., J.P.H. Burt, and R. Pethig, Applications of a new optical technique for measuring the dielectrophoretic behaviour of microorganisms. Biochimica et Biophysica Acta, 1988. 964(2): p. 221-230.

22. Talary, M.S. and R. Pethig, Optical technique for measuring the positive and negative dielectrophoretic behaviour of cells and colloidal suspensions. Iee Proceedings-Science Measurement and Technology, 1994. 141(5): p. 395-399.

23. Bakewell, D.J.G., Nanosize particle movement in time-modulated nonuniform electric fields: a Fourier-Bessel series model. Biomems and Nanotechnology III, 2008. 6799: p. 79914-79914.

24. Markx, G.H., M.S. Talary, and R. Pethig, Separation of Viable and Nonviable Yeast Using Dielectrophoresis. Journal of Biotechnology, 1994. 32(1): p. 29-37.

25. Castellanos, A., et al., Electrohydrodynamics and dielectrophoresis in microsystems: scaling laws. Journal of Physics D-Applied Physics, 2003. 36(20): p. 2584-2597.

26. Bakewell, D.J.G., Dielectrophoresis of colloids and polyelectrolytes, in Electronics and Electrical Engineering. 2002, University of Glasgow: Glasgow. p. 357.

27. Gardiner, C.W., Handbook of stochastic methods for physics, chemistry, and the natural sciences. 1985, Berlin: Springer-Verlag.

28. Haken, H., Synergetics - an introduction. 2nd ed. 1978, Berlin: Springer-Verlag.

29. Gradshteyn, I.S. and I.M. Ryzhik, Table of integrals, series, and products. 5 ed, ed. A. Jeffrey. 1994, London: Academic Press. 
30. Press, W.H., et al., Numerical Recipes. 3rd ed. 2007: C.U.P.

31. Watson, G.N., A treatise on the theory of Bessel functions. . 1952, Cambridge: CUP.

32. Tolstov, G.P., Fourier series. 1962, Englewood Cliffs: Prentice-Hall.

33. Lebedev, N.N., Special functions and their applications., ed. R.A. Silverman. 1965, Englewood Cliffs: PrenticeHall.

34. Abramowitz, M. and I.A. Stegun, Handbook of mathematical functions: Dover, N. Y.

35. Walpole, R.E. and R.H. Myers, Probability and statistics for engineers and scientists. 2nd ed. 1978, New York: Macmillan.

36. Carlson, A.B., P.B. Crilly, and J.C. Rutledge, Communication Systems: An Introduction to Signals and Noise in Electrical Communication. 4th ed. 2002, New York: McGraw Hill. 


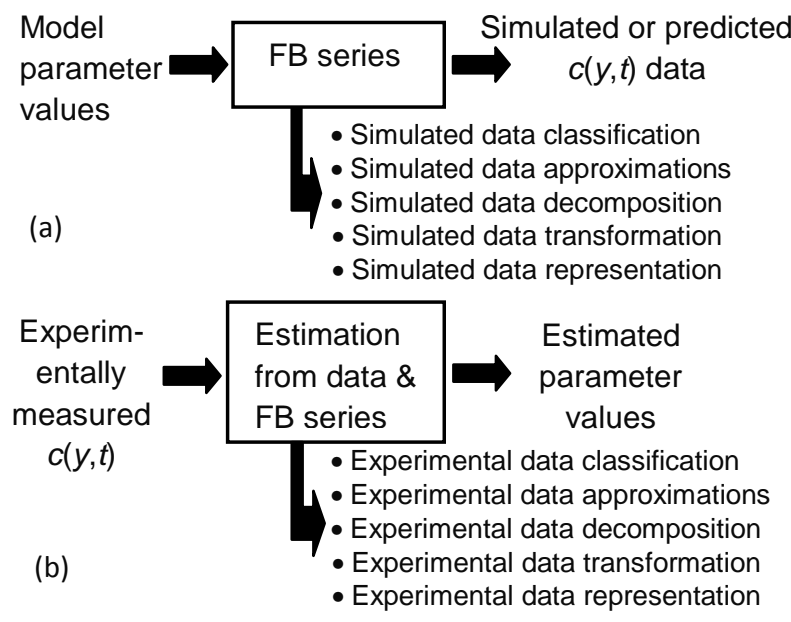




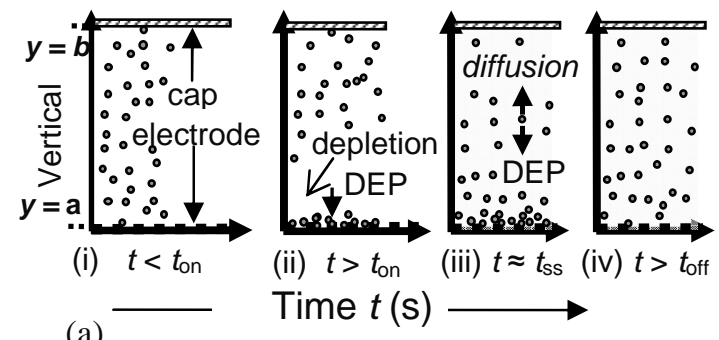

(a) Icollection rate

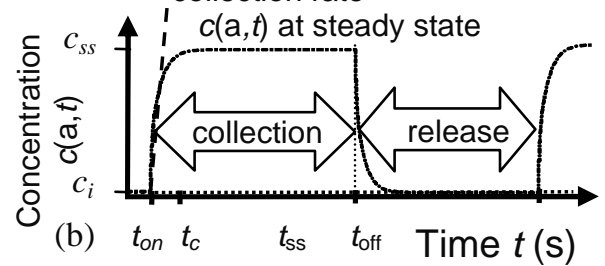




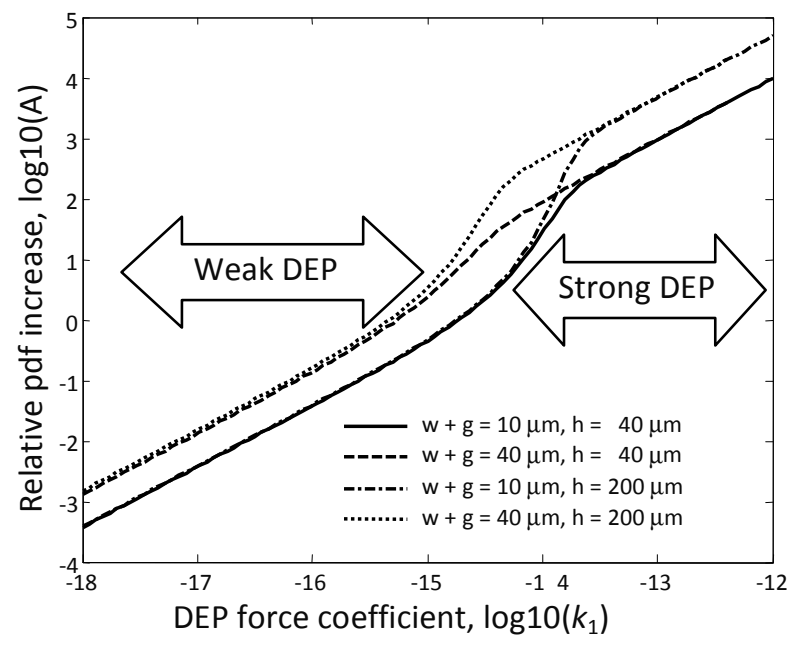



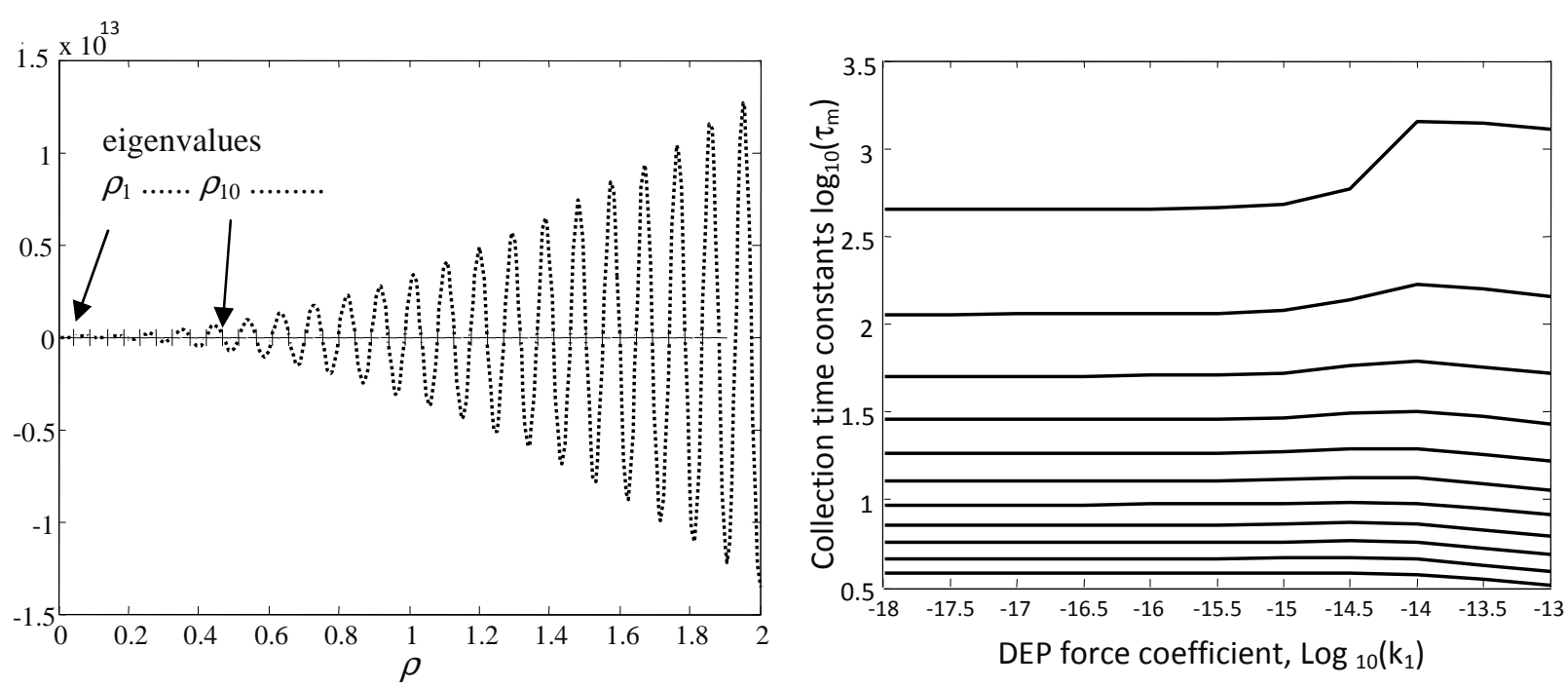


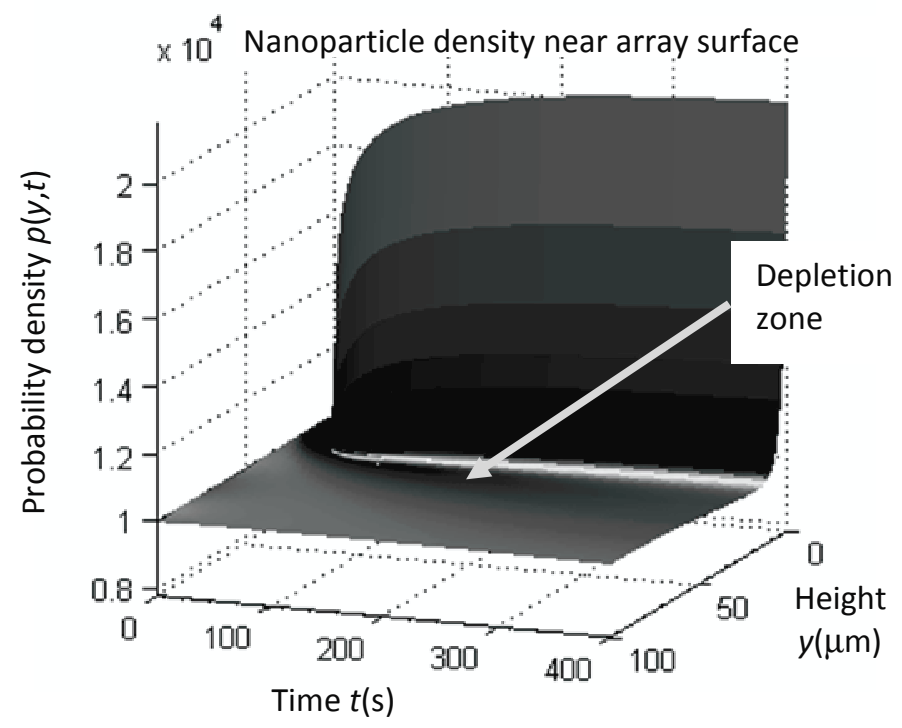



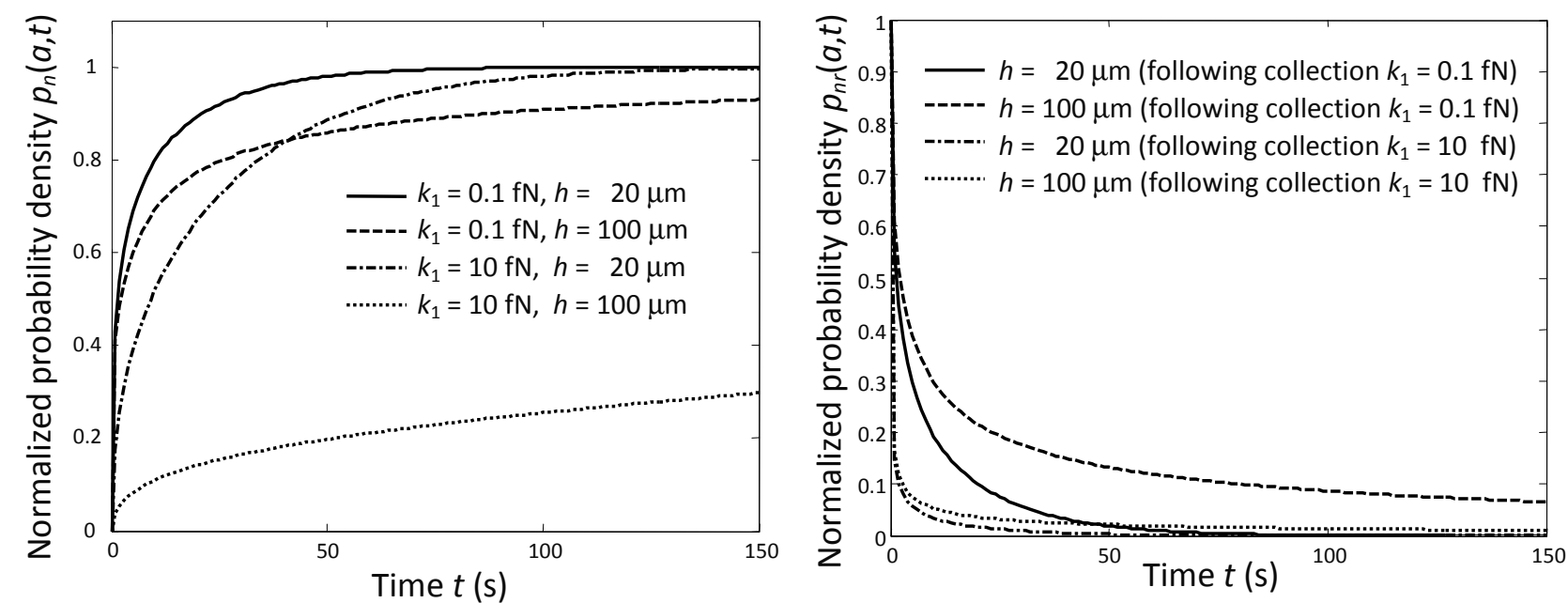

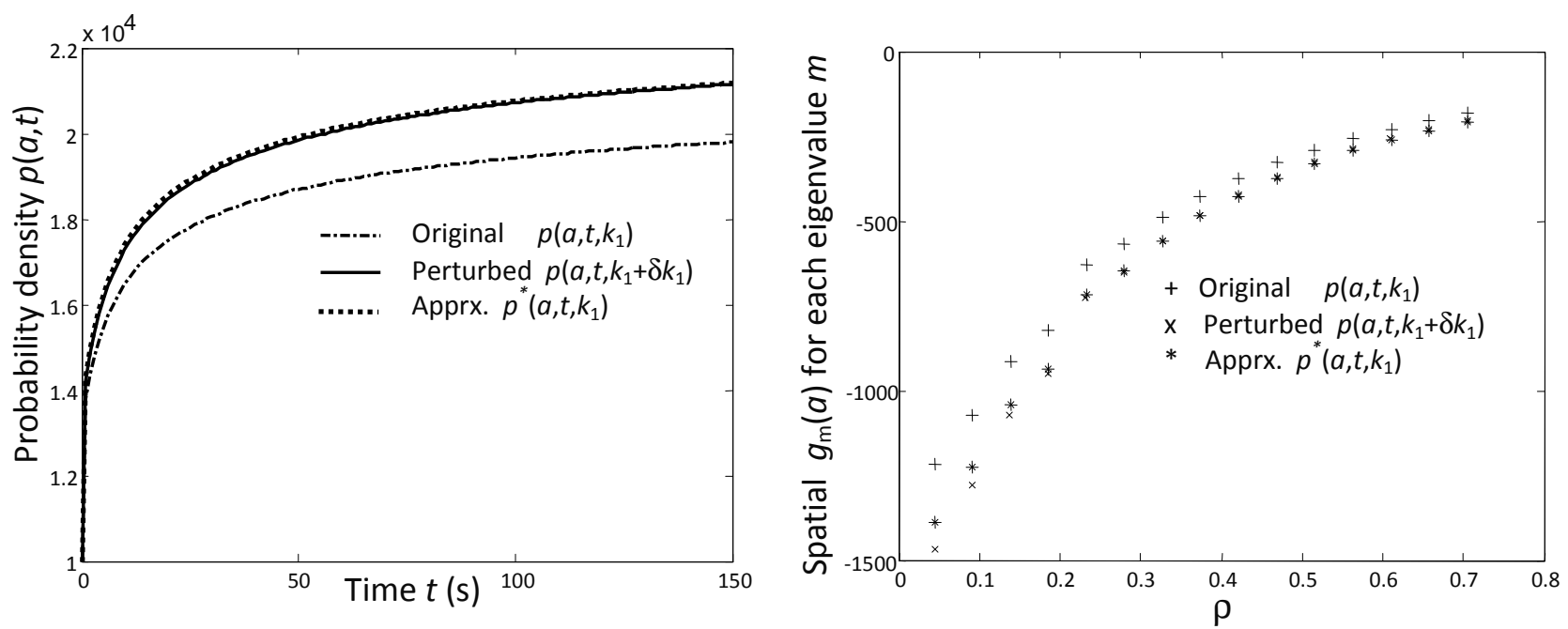


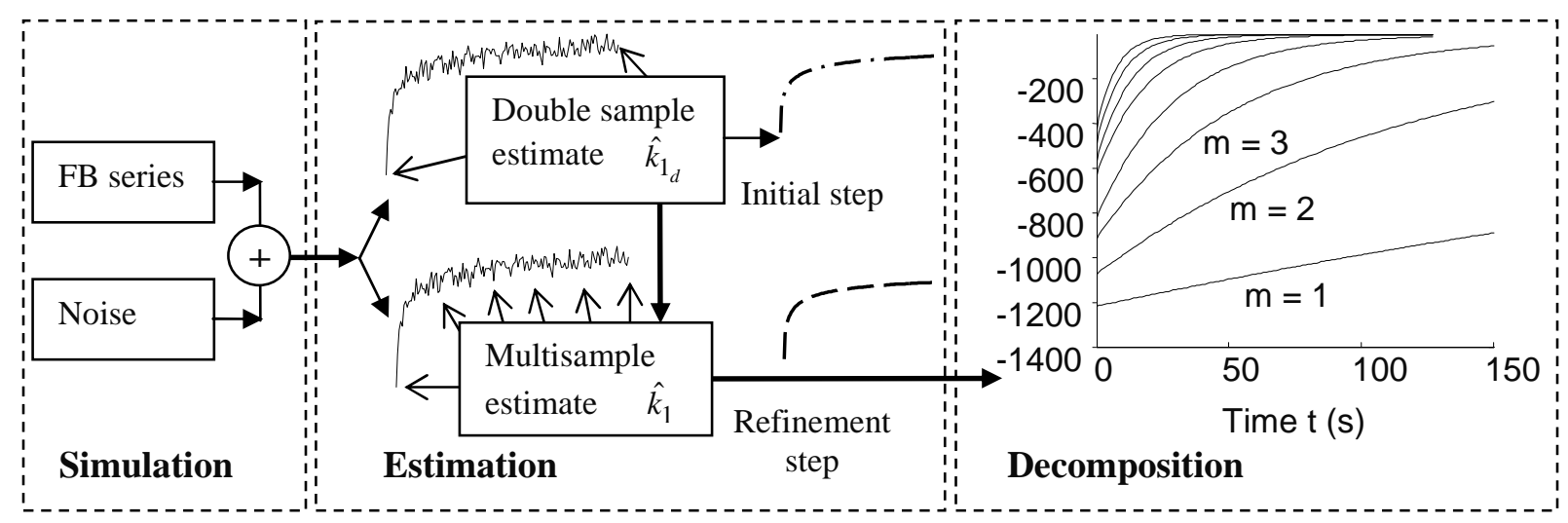



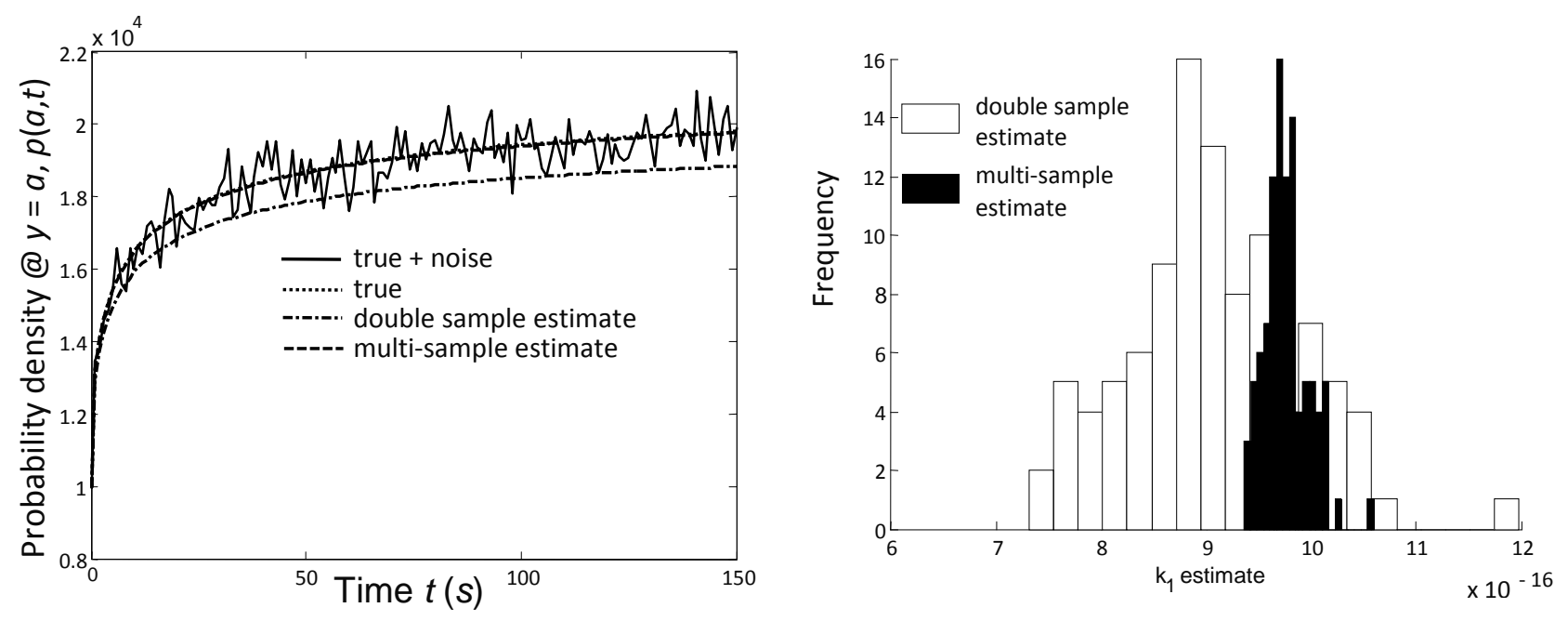


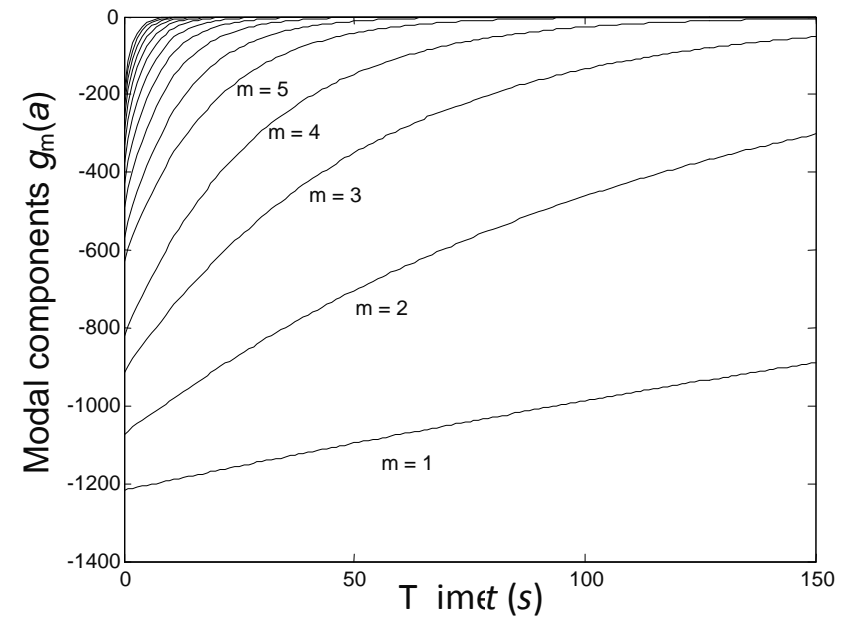




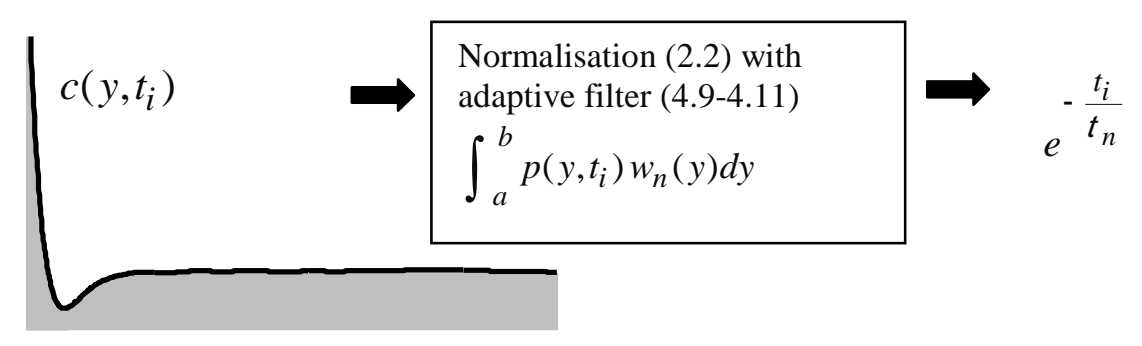




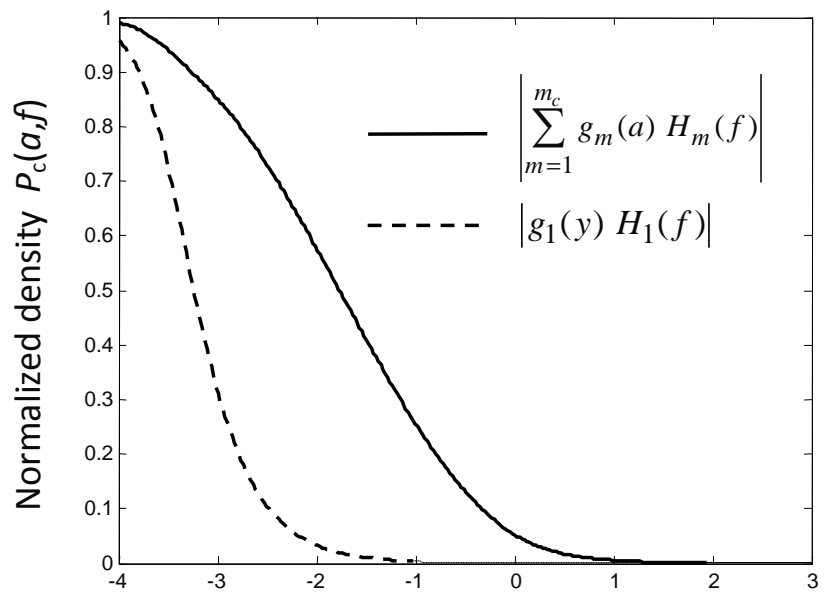

Frequency $\log _{10}(\mathrm{f})(\mathrm{Hz})$ 


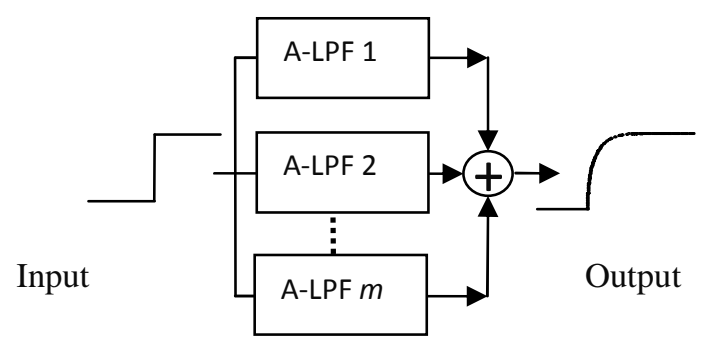

